Trinity University

Digital Commons @ Trinity

Mathematics Faculty Research

Mathematics Department

6-2015

\title{
Connection Coefficients Between Generalized Rising and Falling Factorial Bases
}

Jeffrey Liese

Brian K. Miceli

Trinity University, bmiceli@trinity.edu

Jeffrey Remmel

Follow this and additional works at: https://digitalcommons.trinity.edu/math_faculty

Part of the Mathematics Commons

\section{Repository Citation}

Liese, J., Miceli, B.K., \& Remmel, J. (2015). Connection coefficients between generalized rising and falling factorial bases. Annals of Combinatorics, 19(2), 337-361. doi:10.1007/s00026-015-0268-7

This Article is brought to you for free and open access by the Mathematics Department at Digital Commons @ Trinity. It has been accepted for inclusion in Mathematics Faculty Research by an authorized administrator of Digital Commons @ Trinity. For more information, please contact jcostanz@trinity.edu. 


\title{
Connection coefficients between generalized rising and falling factorial bases
}

\author{
Jeffrey Liese \\ Department of Mathematics \\ California Polytechnic State University \\ San Luis Obispo, CA 93407-0403. USA \\ jliesedcalpoly.edu
}

\author{
Brian K. Miceli \\ Department of Mathematics \\ Trinity University \\ One Trinity Place \\ San Antonio, TX 78212-7200. USA \\ bmiceli@trinity.edu
}

\author{
Jeffrey Remmel* \\ Department of Mathematics \\ University of California, San Diego \\ La Jolla, CA 92093-0112. USA \\ remmel@math.ucsd.edu
}

Submitted: date; Accepted: date;

MR Subject Classifications: 05A15, 05E05

\begin{abstract}
Let $\mathcal{S}=\left(s_{1}, s_{2}, \ldots\right)$ be any sequence of nonnegative integers and let $S_{k}=$ $\sum_{i=1}^{k} s_{i}$ We then define the falling (rising) factorials relative to $\mathcal{S}$ by setting $(x) \downarrow_{k, \mathcal{S}}=$ $\left(x-S_{1}\right)\left(x-S_{2}\right) \cdots\left(x-S_{k}\right)$ and $(x) \uparrow_{k, \mathcal{S}}=\left(x+S_{1}\right)\left(x+S_{2}\right) \cdots\left(x+S_{k}\right)$ if $k \geq 1$ with $(x) \downarrow_{0, \mathcal{S}}=(x) \uparrow_{0, \mathcal{S}}=1$. It follows that $\left\{(x) \downarrow_{k, \mathcal{S}}\right\}_{k \geq 0}$ and $\left\{(x) \uparrow_{k, \mathcal{S}}\right\}_{k \geq 0}$ are bases for the polynomial ring $\mathbb{Q}[x]$. We use a rook theory model due to Miceli and Remmel to give combinatorial interpretations for the connection coefficients between any two of the bases $\left\{(x) \downarrow_{k, \mathcal{S}}\right\}_{k \geq 0},\left\{(x) \uparrow_{k, \mathcal{S}}\right\}_{k \geq 0},\left\{(x) \downarrow_{k, \mathcal{T}}\right\}_{k \geq 0}$, and $\left\{(x) \uparrow_{k, \mathcal{T}}\right\}_{k \geq 0}$ for any two sequences of nonnegative integers $\mathcal{S}=\left(s_{1}, s_{2}, \ldots\right)$ and $\mathcal{T}=\left(t_{1}, t_{2}, \ldots\right)$. We also give two different $q$-analogues of such coefficients. Moreover, we use this rook model to give an alternative combinatorial interpretation of such coefficients in terms of certain pairs of colored permutations and set partitions with restricted insertion patterns.
\end{abstract}

Keywords: rook theory, rook placements, connection coefficients, $q$-analogues, Stirling numbers

\footnotetext{
*Supported in part by NSF grant DMS 0400507
} 


\section{Introduction}

Let $\mathcal{S}=\left(s_{1}, s_{2}, \ldots\right)$ and $\mathcal{T}=\left(t_{1}, t_{2}, \ldots\right)$ be any two sequences of nonnegative integers. For $k \geq 1$, let $S_{k}=\sum_{i=1}^{k} s_{i}$ be the $k^{t h}$ partial sum of the $s_{i}$ 's and $T_{k}=\sum_{i=1}^{k} t_{i}$ be the $k^{\text {th }}$ partial sum of the $t_{i}$ 's. We then define the falling (rising) factorials for $\mathcal{S}$ by setting $(x) \downarrow_{k, \mathcal{S}}=\left(x-S_{1}\right)\left(x-S_{2}\right) \cdots\left(x-S_{k}\right)\left((x) \uparrow_{k, \mathcal{S}}=\left(x+S_{1}\right)\left(x+S_{2}\right) \cdots\left(x+S_{k}\right)\right)$ if $k \geq 1$ and setting $(x) \downarrow_{0, \mathcal{S}}=1\left((x) \uparrow_{0, \mathcal{S}}=1\right)$. These are natural generalizations of the usual falling and rising factorials. For example, if $\mathcal{S}=(0, j, j, j, \ldots)$, then $(x) \downarrow_{n, \mathcal{S}}=x(x-j) \cdots(x-(n-1) j)$ is the usual falling factorial by $j$ and $(x) \uparrow_{n, \mathcal{S}}=x(x+j) \cdots(x+(n-1) j)$ for $n \geq 1$ is the usual rising factorial by $j$. Note that $\left\{(x)_{\downarrow_{k, \mathcal{S}}}\right\}_{k \geq 0},\left\{(x) \uparrow_{k, \mathcal{S}}\right\}_{k \geq 0},\left\{(x) \downarrow_{k, \mathcal{T}}\right\}_{k \geq 0}$, and $\left\{(x) \uparrow_{k, \mathcal{T}}\right\}_{k \geq 0}$ are bases for the polynomial ring $\mathbb{Q}[x]$. In this paper, we shall study the combinatorics of the connection coefficients $r_{n, k}^{\mathcal{T \uparrow}, \mathcal{S} \downarrow}, r_{n, k}^{\mathcal{T} \downarrow, \mathcal{S} \uparrow}, r_{n, k}^{\mathcal{T}, \mathcal{S} \uparrow}$, and $r_{n, k}^{\mathcal{T} \downarrow \mathcal{S} \downarrow}$ defined by the following equations:

$$
\begin{aligned}
(x) \uparrow_{n, \mathcal{T}} & =\sum_{k=0}^{n} r_{n, k}^{\mathcal{T} \uparrow, \mathcal{S} \downarrow}(x) \downarrow_{k, \mathcal{S}}, \\
(x) \downarrow_{n, \mathcal{T}} & =\sum_{k=0}^{n} r_{n, k}^{\mathcal{T} \downarrow, \mathcal{S} \uparrow}(x) \uparrow_{k, \mathcal{S}}, \\
(x) \uparrow_{n, \mathcal{T}} & =\sum_{k=0}^{n} r_{n, k}^{\mathcal{T} \uparrow, \mathcal{S} \uparrow}(x) \uparrow_{k, \mathcal{S}}, \text { and } \\
(x) \downarrow_{n, \mathcal{T}} & =\sum_{k=0}^{n} r_{n, k}^{\mathcal{T} \downarrow \mathcal{S} \downarrow}(x) \downarrow_{k, \mathcal{S}} .
\end{aligned}
$$

More generally, we shall consider two different $q$-analogues of the connection coefficients $r_{n, k}^{\mathcal{T} \uparrow, \mathcal{S} \downarrow}, r_{n, k}^{\mathcal{T} \downarrow, \mathcal{S} \uparrow}, r_{n, k}^{\mathcal{T} \uparrow, \mathcal{S} \uparrow}$, and $r_{n, k}^{\mathcal{T} \downarrow, \mathcal{S} \downarrow}$. That is, there are two natural $q$-analogues of equations (1.1), (1.2), (1.3), and (1.4). First let $[x]_{q}=\frac{1-q^{x}}{1-q}$. If $x$ is a positive integer, then $[x]_{q}=1+q+\cdots+q^{x-1}$. Given a sequence of nonnegative integers $\mathcal{S}=\left(s_{1}, s_{2}, \ldots\right)$, we then let

$$
\begin{aligned}
\langle x\rangle_{q} \downarrow_{k, \mathcal{S}} & =\left([x]_{q}-\left[S_{1}\right]_{q}\right)\left([x]_{q}-\left[S_{2}\right]_{q}\right) \cdots\left([x]_{q}-\left[S_{k}\right]_{q}\right) \text { and } \\
\langle x\rangle_{q} \uparrow_{k, \mathcal{S}} & =\left([x]_{q}+\left[S_{1}\right]_{q}\right)\left([x]_{q}+\left[S_{2}\right]_{q}\right) \cdots\left([x]_{q}+\left[S_{k}\right]_{q}\right)
\end{aligned}
$$

if $k \geq 1$, and we let $\langle x\rangle_{q} \downarrow_{0, \mathcal{S}}=\langle x\rangle_{q} \uparrow_{0, \mathcal{S}}=1$. Here, $\left\{\langle x\rangle_{q} \downarrow_{n, \mathcal{S}}\right\}_{k \geq 0}$ and $\left\{\langle x\rangle_{q} \uparrow_{n, \mathcal{S}}\right\}_{k \geq 0}$ are bases for the polynomial ring $\mathbb{Q}(q)\left[q^{x}\right]$. We define our first $q$-analogue of the connection coefficients $r_{n, k}^{\mathcal{T \uparrow}, \mathcal{S} \downarrow}, r_{n, k}^{\mathcal{T \downarrow} \backslash, \mathcal{S} \uparrow}, r_{n, k}^{\mathcal{T}, \mathcal{S} \uparrow}$, and $r_{n, k}^{\mathcal{T \downarrow}, \mathcal{S} \downarrow}$ by the following equations: 


$$
\begin{aligned}
\langle x\rangle_{q} \uparrow_{n, \mathcal{T}} & =\sum_{k=0}^{n} r_{n, k}^{\mathcal{T} \uparrow, \mathcal{S} \downarrow}(q)\langle x\rangle_{q \downarrow} \downarrow_{k, \mathcal{S}}, \\
\langle x\rangle_{q} \downarrow_{n, \mathcal{T}} & =\sum_{k=0}^{n} r_{n, k}^{\mathcal{T} \downarrow, \mathcal{S} \uparrow}(q)\langle x\rangle_{q} \uparrow_{k, \mathcal{S}}, \\
\langle x\rangle_{q} \uparrow_{n, \mathcal{T}} & =\sum_{k=0}^{n} r_{n, k}^{\mathcal{T} \uparrow, \mathcal{S} \uparrow}(q)\langle x\rangle_{q} \uparrow_{k, \mathcal{S}}, \text { and } \\
\langle x\rangle_{q} \downarrow_{n, \mathcal{T}} & =\sum_{k=0}^{n} r_{n, k}^{\mathcal{T} \downarrow, \mathcal{S} \downarrow}(q)\langle x\rangle_{q} \downarrow_{k, \mathcal{S}} .
\end{aligned}
$$

Similarly, we let

$$
\begin{aligned}
& {[x]_{q} \downarrow_{k, \mathcal{S}}=\left(\left[x-S_{1}\right]_{q}\right)\left(\left[x-S_{2}\right]_{q}\right) \cdots\left(\left[x-S_{k}\right]_{q}\right) \text { and }} \\
& {[x]_{q} \uparrow_{k, \mathcal{S}}=\left(\left[x+S_{1}\right]_{q}\right)\left(\left[x+S_{2}\right]_{q}\right) \cdots\left(\left[x+S_{k}\right]_{q}\right)}
\end{aligned}
$$

if $k \geq 1$ and let $[x]_{q} \downarrow_{0, \mathcal{S}}=[x]_{q} \uparrow_{0, \mathcal{S}}=1$. We define our second $q$-analogue of the connection coefficients $r_{n, k}^{\mathcal{T} \uparrow, \mathcal{S} \downarrow}, r_{n, k}^{\mathcal{T} \downarrow, \mathcal{S} \uparrow}, r_{n, k}^{\mathcal{T} \uparrow, \mathcal{S} \uparrow}$, and $r_{n, k}^{\mathcal{T} \downarrow, \mathcal{S} \downarrow}$ by the following equations:

$$
\begin{aligned}
{[x]_{q} \uparrow_{n, \mathcal{T}} } & =\sum_{k=0}^{n} \tilde{r}_{n, k}^{\mathcal{T}, \mathcal{S} \downarrow}(q)[x]_{q} \downarrow_{k, \mathcal{S}}, \\
{[x]_{q} \downarrow_{n, \mathcal{T}} } & =\sum_{k=0}^{n} \tilde{r}_{n, k}^{\mathcal{T} \downarrow, \mathcal{S} \uparrow}(q)[x]_{q} \uparrow_{k, \mathcal{S}}, \\
{[x]_{q} \uparrow_{n, \mathcal{T}} } & =\sum_{k=0}^{n} \tilde{r}_{n, k}^{\mathcal{T} \uparrow, \mathcal{S} \uparrow}(q)[x]_{q} \uparrow_{k, \mathcal{S}}, \text { and } \\
{[x]_{q} \downarrow_{n, \mathcal{T}} } & =\sum_{k=0}^{n} \tilde{r}_{n, k}^{\mathcal{T} \downarrow, \mathcal{S} \downarrow}(q)[x]_{q} \downarrow_{k, \mathcal{S}} .
\end{aligned}
$$

The main goal of this paper is to give combinatorial interpretations of the connection coefficients $r_{n, k}^{\mathcal{T \uparrow}, \mathcal{S} \downarrow}(q), r_{n, k}^{\mathcal{T} \downarrow, \mathcal{S} \uparrow}(q), r_{n, k}^{\mathcal{T} \uparrow, \mathcal{S} \uparrow}(q)$, and $r_{n, k}^{\mathcal{T} \downarrow, \mathcal{S} \downarrow}(q)$ for all $0 \leq k \leq n$. We shall see that a rook theory model of Miceli and Remmel [10] provides a setting where we can essentially give a uniform combinatorial interpretation of these connection coefficients. We shall also show that a slight variation of that model will also allow us to give combinatorial interpretations of the connection coefficients $\tilde{r}_{n, k}^{\mathcal{T} \uparrow, \mathcal{S} \downarrow}(q), \tilde{r}_{n, k}^{\mathcal{T} \downarrow, \mathcal{S} \uparrow}(q), \tilde{r}_{n, k}^{\mathcal{T} \uparrow, \mathcal{S} \uparrow}(q)$, and $\tilde{r}_{n, k}^{\mathcal{T} \downarrow, \mathcal{S} \downarrow}(q)$.

We should note that in certain special cases, rook theory interpretations of the coefficients $\tilde{r}_{n, k}^{\mathcal{T} \uparrow, \mathcal{S} \downarrow}(q), \tilde{r}_{n, k}^{\mathcal{T} \downarrow, \mathcal{S} \uparrow}(q), \tilde{r}_{n, k}^{\mathcal{T}, \mathcal{S} \uparrow}(q)$, and $\tilde{r}_{n, k}^{\mathcal{T} \downarrow, \mathcal{S} \downarrow}(q)$ have appeared in the literature. For example, a Ferrers board $B=F\left(b_{1}, \ldots, b_{n}\right)$ is a subset of an $n \times m$ chessboard such that for $i=1, \ldots, n$, the lowest $b_{i}$ squares are in $B$ and $0 \leq b_{1} \leq \cdots \leq b_{n}$. Garsia 
and Remmel [3] defined $q$-analogues of rook numbers $r_{n-k}(B, q)$ for Ferrers boards $B=F\left(b_{1}, \ldots, b_{n}\right)$ and gave a rook theory proof of the identity

$$
\prod_{i=1}^{n}\left[x+b_{i}-(i-1)\right]_{q}=\sum_{k=0}^{n} r_{n-k}(B, q)[x]_{q}[x-1]_{q} \cdots[x-(k-1)]_{q} .
$$

Remmel and Wachs [12] defined a more restricted class of $q$-analogues of rook numbers, $\tilde{r}_{k}^{j}(B, q)$, in their $j$-attacking rook model and proved that for Ferrers boards $B=$ $F\left(b_{1}, \ldots, b_{n}\right)$, where $b_{i+1}-b_{i} \geq j-1$ if $b_{i} \neq 0$,

$$
\prod_{i=1}^{n}\left[x+b_{i}-j(i-1)\right]_{q}=\sum_{k=0}^{n} \tilde{r}_{n-k}^{j}(B, q)[x]_{q}[x-j]_{q}[x-2 j]_{q} \cdots[x-(k-1) j]_{q} .
$$

Goldman and Haglund [4] developed an $i$-creation rook theory model and an appropriate notion of rook numbers $r_{n-k}^{(i)}(B, q)$ for which the following identity holds for Ferrers boards:

$$
\prod_{j=1}^{n}\left[x+b_{j}+(j-1)(i-1)\right]_{q}=\sum_{k=0}^{n} r_{n-k}^{(i)}(B, q)[x]_{q}[x+(i-1)]_{q} \cdots[x+(k-1)(i-1)]_{q} .
$$

Thus, in the situation where the right-hand sides involve either rising factorials by $j$ $[x]_{q}[x+j]_{q} \cdots[x+(k-1) j]_{q}$ - or falling factorials by $j-[x]_{q}[x-j]_{q} \cdots[x-(k-1) j]_{q}$ - certain special cases of the coefficients $\tilde{r}_{n, k}^{\mathcal{T}, \mathcal{S} \downarrow}(q), \tilde{r}_{n, k}^{\mathcal{T} \downarrow, \mathcal{S} \uparrow}(q), \tilde{r}_{n, k}^{\mathcal{T}, \mathcal{S} \uparrow}(q)$, and $\tilde{r}_{n, k}^{\mathcal{T} \downarrow, \mathcal{S} \downarrow}(q)$ have been given rook theory interpretations. These include various $q$-analogues of the Stirling numbers of the first and second kind - see [1], [2], [3], [5], [7], [8], [9], [11], [13], [14], [15] and two $q$-analogues of the Lah numbers - see [3], [6]. However, none of these papers consider $q$-analogues of the more general rising and falling factorial basis $\left\{(x)_{k, \mathcal{S}}\right\}_{k \geq 0}$ and $\left\{(x) \uparrow_{k, \mathcal{S}}\right\}_{k \geq 0}$.

The outline of this paper is as follows. In section 2, we shall describe the augmented rook model due to Miceli and Remmel [10]. In section 3, we shall use the MiceliRemmel rook model to give direct combinatorial interpretations of $r_{n, k}^{\mathcal{T \uparrow}, \mathcal{S} \downarrow}(q), r_{n, k}^{\mathcal{T} \downarrow, \mathcal{S} \uparrow}(q)$, $r_{n, k}^{\mathcal{T} \uparrow \mathcal{S} \uparrow}(q)$, and $r_{n, k}^{\mathcal{T} \downarrow \mathcal{S} \downarrow}(q)$. As $r_{n, k}^{\mathcal{T} \uparrow, \mathcal{S} \downarrow}(1)=r_{n, k}^{\mathcal{T}, \mathcal{S} \downarrow}, r_{n, k}^{\mathcal{T} \downarrow, \mathcal{S} \uparrow}(1)=r_{n, k}^{\mathcal{T} \downarrow, \mathcal{S} \uparrow}, r_{n, k}^{\mathcal{T} \uparrow, \mathcal{S} \uparrow}(1)=r_{n, k}^{\mathcal{T} \uparrow, \mathcal{S} \uparrow}$, and $r_{n, k}^{\mathcal{T} \downarrow \mathcal{S} \downarrow}(1)=r_{n, k}^{\mathcal{T} \downarrow, \mathcal{S} \downarrow}$, we will automatically obtain combinatorial interpretations of $r_{n, k}^{\mathcal{T}, \mathcal{S} \downarrow}$, $r_{n, k}^{\mathcal{T} \downarrow, \mathcal{S} \uparrow}, r_{n, k}^{\mathcal{T} \uparrow, \mathcal{S} \uparrow}$, and $r_{n, k}^{\mathcal{T} \downarrow \mathcal{S} \downarrow}$. Subsequently, we shall modify the aforementioned interpretations to obtain combinatorial interpretations of $\tilde{r}_{n, k}^{\mathcal{T} \uparrow \mathcal{S} \downarrow}(q), \tilde{r}_{n, k}^{\mathcal{T} \downarrow \mathcal{S} \uparrow}(q), \tilde{r}_{n, k}^{\mathcal{T} \uparrow \mathcal{S} \uparrow}(q)$, and $\tilde{r}_{n, k}^{\mathcal{T} \downarrow \mathcal{S} \downarrow}(q)$. Lastly, in section 4 we shall give alternative combinatorial interpretations of $r_{n, k}^{\mathcal{T} \uparrow, \mathcal{S} \downarrow}(q)$, $r_{n, k}^{\mathcal{T} \downarrow \mathcal{S} \uparrow}(q), r_{n, k}^{\mathcal{T} \uparrow \mathcal{S} \uparrow}(q)$, and $r_{n, k}^{\mathcal{T} \downarrow \mathcal{S} \downarrow}(q)$ in terms of certain pairs, $(\sigma, \pi)$, where $\sigma$ is a colored permutation of $\{1,2, \ldots, n\}$ with certain restricted insertion patterns and $\pi$ is a colored set partition of $\{1,2, \ldots, n\}$ with certain restricted insertion patterns.

\section{The Miceli-Remmel Rook Theory Model}

Let $\mathbb{N}_{0}=\{0,1,2, \ldots\}$, and let $[n]:=\{1,2, \ldots, n\}$ for any positive integer $n$. We let $B_{n, m}=[n] \times[m]$ be an $n$ by $m$ array of squares. We number the rows of $B_{n, m}$ from 


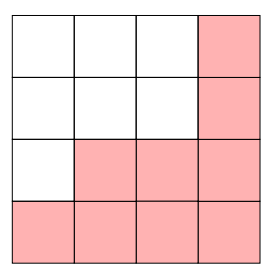

Figure 1: A Ferrers board $B=F(1,2,2,4) \subseteq B_{4,4}$.

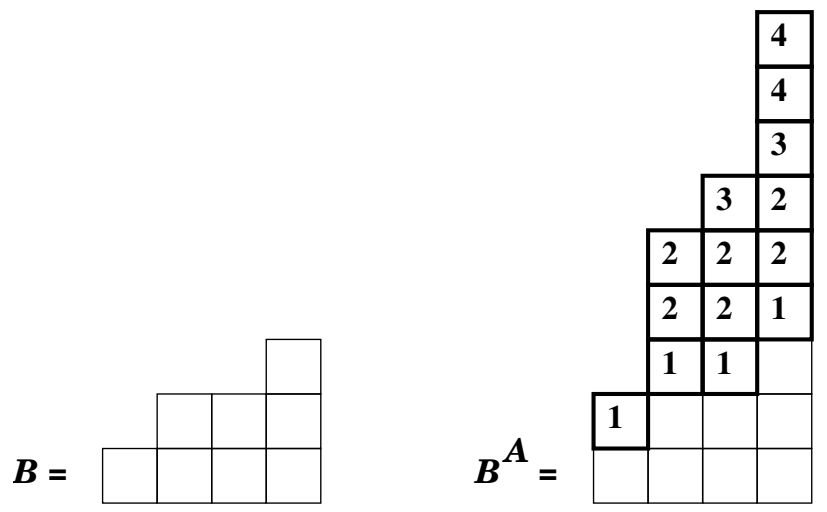

Figure 2: The Ferrers board $B$ with $\mathcal{B}=(1,2,2,3)$ and the augmented rook board, $\mathcal{B}^{\mathcal{A}}$, with $\mathcal{B}=(1,2,2,3)$ and $\mathcal{A}=(1,2,1,2)$.

bottom to top with the numbers $1, \ldots, m$ and the columns of $B_{n, m}$ from left to right with the numbers $1, \ldots, n$ and refer to the square or cell in the $i^{\text {th }}$ row and $j^{\text {th }}$ column of $B_{n, m}$ as the $(i, j)^{t h}$ cell of $B_{n, m}$. A rook board $B$ is any subset of $B_{n, m}$. If $B \subseteq B_{n, m}$ is a rook board consisting of the first $b_{i}$ cells of column $i$ for $i=1, \ldots, n$, then we will write $B=F\left(b_{1}, \ldots, b_{n}\right)$ and refer to $B$ as a skyline board. In the special case that $0 \leq b_{1} \leq b_{2} \leq \cdots \leq b_{n} \leq m$, we will say that $B=F\left(b_{1}, b_{2}, \ldots, b_{n}\right)$ is a Ferrers board. For example, $F(1,2,2,4)$ is a Ferrers board and is pictured in Figure 1.

Suppose that we are given two sequences from $\mathbb{N}_{0}^{n}, \mathcal{B}=\left\{b_{i}\right\}_{i=1}^{n}$ and $\mathcal{A}=\left\{a_{i}\right\}_{i=1}^{n}$. We let $A_{i}=a_{1}+a_{2}+\cdots+a_{i}$ be the $i^{t h}$ partial sum of the $a_{i}$ 's and $B=F\left(b_{1}, b_{2}, \ldots, b_{n}\right)$. We will consider the augmented rook board, $\mathcal{B}^{\mathcal{A}}$, which is constructed by starting with the board $B$ and then adding $A_{i}$ cells on top of the $i^{\text {th }}$ column for $i=1, \ldots, n$. Thus $\mathcal{B}^{\mathcal{A}}$ can be thought of as the board $F\left(b_{1}+A_{1}, b_{2}+A_{2}, \ldots, b_{n}+A_{n}\right)$. For example, if $\mathcal{B}=(1,2,2,3)$ and $\mathcal{A}=(1,2,1,2)$, then Figure 2 pictures the board $B$ and the board $\mathcal{B}^{\mathcal{A}}$. We will refer to the part of the board consisting of the $b_{i}$ 's as the base part of $\mathcal{B}^{\mathcal{A}}$ and the part which corresponds to the $a_{i}{ }^{\prime}$ 's as the augmented part of $\mathcal{B}^{\mathcal{A}}$. Moreover, for each column $i$, we will refer to the cells in rows $b_{i}+1, \ldots, b_{i}+a_{1}$ as the $a_{1}^{\text {th }}$ part of $i^{\text {th }}$ column, the cells in row $b_{i}+a_{1}+1, \ldots, b_{i}+a_{1}+a_{2}$ as the $a_{2}^{n d}$ part of $i^{\text {th }}$ column, and so on in this fashion. In Figure 2, we have indicated the $a_{j}^{\text {th }}$ part of each column by putting a $j$ in those cells.

Next we must define the appropriate notion of non-attacking rook placements in $\mathcal{B}^{\mathcal{A}}$. We first consider placements $\mathbb{P}$ of rooks in $\mathcal{B}^{\mathcal{A}}$ where there is at most one rook in each column. The leftmost rook of $\mathbb{P}$ will cancel all the cells in the columns to its right 


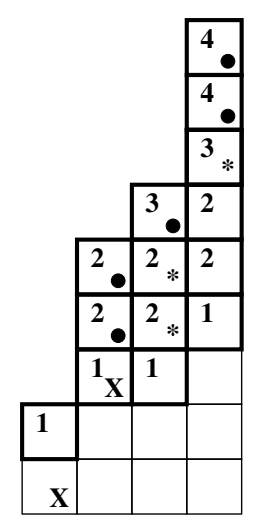

Figure 3: A placement of two rooks in an augmented rook board, $\mathcal{B}^{\mathcal{A}}$, with $\mathcal{B}=$ $(1,2,2,3)$ and $\mathcal{A}=(1,2,1,2)$.

which correspond to the $a_{j}^{\text {th }}$ part of that column of highest index. Thus, if the leftmost rook is in column $i$, then it will cancel the $a_{j}^{\text {th }}$ part of column $j$ for $j=i+1, \ldots, n$. In general, each rook will cancel all the cells in the columns to its right which correspond to the $a_{j}^{\text {th }}$ part of that column where $j$ is the highest index such that the cells of $a_{j}^{\text {th }}$ part of the column have not been canceled by any rook to its left. We then let $\mathcal{N}_{k}\left(\mathcal{B}^{\mathcal{A}}\right)$ denote the set of placements of $k$ rooks in the board $\mathcal{B}^{\mathcal{A}}$ such that

1. there is at most one rook per column, and

2. no rook lies in a cell which has been canceled by a rook to its left.

For example, if $\mathcal{B}=(1,2,2,3)$ and $\mathcal{A}=(1,2,1,2)$, then we have illustrated in Figure 3 a placement $\mathbb{P} \in \mathcal{N}_{2}\left(\mathcal{B}^{\mathcal{A}}\right)$ where each rook is denoted by an " $\mathrm{X}$ " and we have placed a "•" in all the cells canceled by the rook in column 1 and a " $*$ " in all the cells canceled by the rook in column 2 . We shall refer to a placement $\mathbb{P} \in \mathcal{N}_{k}\left(\mathcal{B}^{\mathcal{A}}\right)$ as a placement of $k$ non-attacking rooks in $\mathcal{B}^{\mathcal{A}}$.

Miceli and Remmel also defined a general augmented rook board $\mathcal{B}_{x}^{\mathcal{A}}$. Given two sequences of nonnegative integers $\mathcal{B}$ and $\mathcal{A}$ and a nonnegative integer $x$, the board $\mathcal{B}_{x}^{\mathcal{A}}$ will have three parts. First we start with the board $\mathcal{B}^{\mathcal{A}}$ which we will refer to as the upper part of $\mathcal{B}_{x}^{\mathcal{A}}$. Here the part of the upper part that corresponds to the board $B=F\left(b_{1}, b_{2}, \ldots, b_{n}\right)$ will be called the base part of $\mathcal{B}_{x}^{\mathcal{A}}$ and the part which corresponds to the $a_{i}$ 's will be called the upper augmented part of $\mathcal{B}_{x}^{\mathcal{A}}$. Directly below $\mathcal{B}^{\mathcal{A}}$, we will attach $x$-rows of length $n$ which will be referred to as the $x$-part of $\mathcal{B}_{x}^{\mathcal{A}}$. Finally, directly below the $x$-part, we will place the flip of the Ferrers board $F\left(A_{1}, \ldots, A_{n}\right)$ which will be called the lower augmented part of $\mathcal{B}_{x}^{\mathcal{A}}$. We will say that $x$-part is separated from the upper part of $\mathcal{B}_{x}^{\mathcal{A}}$ by the high bar and from the lower augmented part of $\mathcal{B}_{x}^{\mathcal{A}}$ by the low bar. For example, Figure 4 pictures the board $\mathcal{B}_{x}^{\mathcal{A}}$ where $\mathcal{B}=(1,2,2,4), \mathcal{A}=(2,1,2,1)$, and $x=4$. Much like we did for the upper augmented part of $\mathcal{B}_{x}^{\mathcal{A}}$, we will refer to the first $a_{1}$ cells of the lower augmented part in column $i$, reading from top to bottom, as the $a_{1}^{s t}$ part of the $i^{t h}$ column of the lower augmented part, the next $a_{2}$ cells, reading from top to bottom, as the $a_{2}^{n d}$ part of the $i^{\text {th }}$ column of the lower augmented part, etc.. 
We now define the set of placements of $n$ non-attacking rooks for the board $\mathcal{B}_{x}^{\mathcal{A}}$. We will consider placements of $n$ rooks on $\mathcal{B}_{x}^{\mathcal{A}}$ where there is exactly one rook in each column. The cancelation rules for each rook are the following:

1. A rook placed above the high bar in the $i^{\text {th }}$ column of $\mathcal{B}_{x}^{\mathcal{A}}$ will cancel all of the cells in columns $i+1, i+2, \ldots, n$, in both the upper and lower augmented parts, which belong to the $a_{j}^{\text {th }}$ part of highest subscript in that column which are not canceled by a rook to the left of column $i$.

2. Rooks placed below the high bar do not cancel anything.

We then let $\mathcal{N}_{n}\left(\mathcal{B}_{x}^{\mathcal{A}}\right)$ denote the set of all placements of $n$ rooks in $\mathcal{B}_{x}^{\mathcal{A}}$ for which there is exactly one rook in each column and no rook lies in a cell which is canceled by a rook to its left. An example of a placement $\mathbb{P} \in \mathcal{N}_{n}\left(\mathcal{B}_{x}^{\mathcal{A}}\right)$ can be seen in the righthand side Figure 4 . Here we have indicated the cells canceled by the rook in the first column by placing a "•" in those cells and the cells canceled by the rook in the third column by placing an " $*$ " in those cells. The rooks placed in the second and fourth columns do not cancel any cells since they are placed below the high bar.

For any positive integer $n$, define $[-n]_{q}:=-[n]_{q}$ where $[n]_{q}=1+q+q^{2}+\cdots+q^{n-1}$, and define $[0]_{q}=0$. Now fix two sequences $\mathcal{B}=\left(b_{1}, \ldots, b_{n}\right)$ and $\mathcal{A}=\left(a_{1}, \ldots, a_{n}\right)$ and two sign functions Sgn $:[n] \rightarrow\{1,-1\}$ and $\overline{\operatorname{Sgn}}:[n] \rightarrow\{1,-1\}$. Let $\bar{A}_{i}:=$ $\sum_{j=1}^{i} \overline{\operatorname{Sgn}}(j) a_{j}$.

Given this terminology, Miceli and Remmel define the $q$-weight, $\mu_{q, \mathcal{B}_{x}^{A}}^{\mathrm{Sgn}}(\mathrm{Sg})$, of each cell $c$ in $\mathcal{B}_{x}^{\mathcal{A}}$, and for the exact weighting scheme, we refer the reader to [10]. From there, the $q$-weight of a placement on the general augmented board $\hat{\mathbb{P}} \in \mathcal{N}_{n}\left(\mathcal{B}_{x}^{\mathcal{A}}\right)$, which has rooks in cells $c_{1}, \ldots, c_{n}$, is given by

$$
\mu_{q, \mathcal{B}_{x}^{A}}^{\mathrm{Sgn}, \overline{\mathrm{Sgn}}}(\hat{\mathbb{P}})=\prod_{i=1}^{n} \mu_{q, \mathcal{B}_{x}^{A}}^{\mathrm{Sgn}, \overline{\mathrm{Sgn}}}\left(c_{i}\right) .
$$

By passing this $q$-weight to the underlying augmented board, the $q$-weight of a placement on the augmented board $\mathbb{P} \in \mathcal{N}_{k}\left(\mathcal{B}^{\mathcal{A}}\right)$, which has rooks in cells $c_{1}, \ldots, c_{k}$, is given by

$$
\mu_{q, \mathcal{B}^{\mathcal{A}}}^{\mathrm{Sgn}, \overline{\operatorname{Sgn}}}(\mathbb{P})=\prod_{i=1}^{k} \mu_{q, \mathcal{B}_{x}^{\mathcal{A}}}^{\mathrm{Sgg}}\left(\overline{\mathrm{Sn}}\left(c_{i}\right) .\right.
$$

Using this $q$-weight of placements in an augmented board, we obtain the definition of the $q$-augmented rook numbers:

$$
r_{k}\left(\mathcal{B}^{\mathcal{A}}, \operatorname{Sgn}, \overline{\mathrm{Sgn}}, q\right)=\sum_{\mathbb{P} \in \mathcal{N}_{k}\left(\mathcal{B}^{\mathcal{A}}\right)} \mu_{q, \mathcal{B}^{\mathcal{A}}}^{\mathrm{Sggn}}(\mathbb{P}) .
$$

An example of the $q$-weights of the cells in $\mathcal{B}_{x}^{\mathcal{A}}$ is given on the left-hand side of Figure 4 in the case where $x=4, \mathcal{B}=(1,2,2,4), \mathcal{A}=(2,1,2,1)$,

$$
\operatorname{Sgn}(i)= \begin{cases}+1 & \text { if } i=1,2,4 \\ -1 & \text { if } i=3\end{cases}
$$



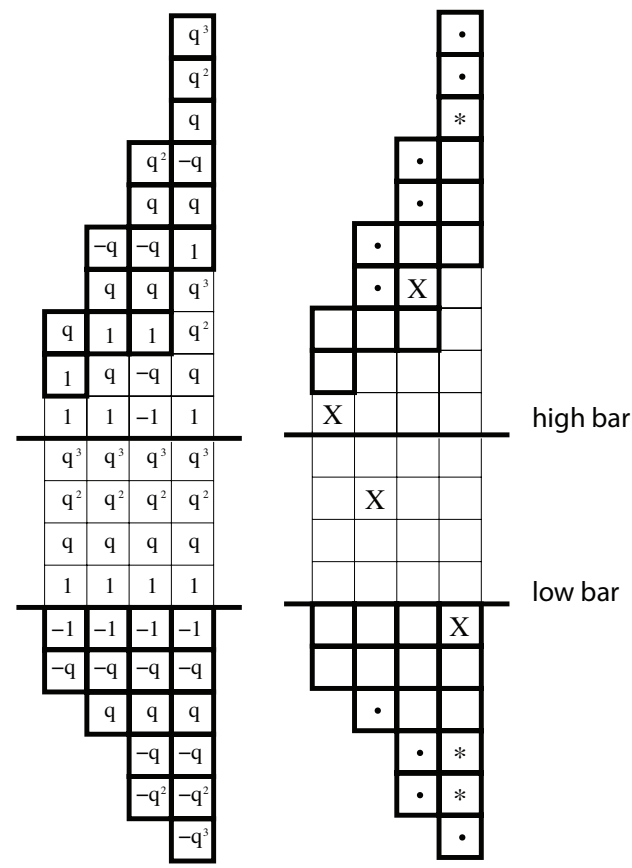

Figure 4: The weighting of cells in placements in $\mathcal{B}_{x}^{\mathcal{A}}$

and

$$
\overline{\operatorname{Sgn}}(i)= \begin{cases}+1 & \text { if } i=2, \\ -1 & \text { if } i=1,3,4 .\end{cases}
$$

If we consider the placement, $\mathbb{P}$, pictured on the right-hand side of Figure 4 , then the rooks are placed in cells with $q$-weights $1, q^{2}, q,-1$, reading from left to right. Thus

$$
\mu_{q, \mathcal{B}_{x}^{\mathcal{A}}}^{\mathrm{Sgn}} \overline{\mathrm{Sgn}}(\mathbb{P})=(1)\left(q^{2}\right)(q)(-1)=-q^{3} .
$$

Finally, Miceli and Remmel proved the following $q$-theorem by $q$-counting placements in $\mathcal{N}_{n}\left(\mathcal{B}_{x}^{\mathcal{A}}\right)$ in two ways. For the details of this proof, we again refer the reader to Theorem 4.1 in [10]. Slight modifications of that proof will be used when altering our combinatorial interpretations of $r_{n, k}^{\mathcal{T} \uparrow \mathcal{S} \downarrow}(q), r_{n, k}^{\mathcal{T} \downarrow, \mathcal{S} \uparrow}(q), r_{n, k}^{\mathcal{T}, \mathcal{S} \uparrow}(q)$, and $r_{n, k}^{\mathcal{T} \downarrow, \mathcal{S} \downarrow}(q)$ to produce our combinatorial interpretations of $\tilde{r}_{n, k}^{\mathcal{T} \uparrow \mathcal{S} \downarrow}(q), \tilde{r}_{n, k}^{\mathcal{T} \downarrow, \mathcal{S}}(q), \tilde{r}_{n, k}^{\mathcal{T}^{\top}, \mathcal{S} \uparrow}(q)$, and $\tilde{r}_{n, k}^{\mathcal{T} \downarrow \mathcal{S} \downarrow}(q)$.

Theorem 2.1. Let $\mathcal{B}=\left\{b_{i}\right\}_{i=1}^{n}$ and $\mathcal{A}=\left\{a_{i}\right\}_{i=1}^{n}$ be any two sequences from $\mathbb{N}_{0}^{n}$ and let Sgn, $\overline{\operatorname{Sgn}}:[n] \rightarrow\{-1,1\}$. Then

$$
\prod_{i=1}^{n}\left([x]_{q}+\operatorname{Sgn}(i)\left[b_{i}\right]_{q}\right)=\sum_{k=0}^{n} r_{n-k}\left(\mathcal{B}^{\mathcal{A}}, \operatorname{Sgn}, \overline{\operatorname{Sgn}}, q\right) \prod_{s=1}^{k}\left([x]_{q}+\left[\bar{A}_{s}\right]_{q}\right) .
$$




\section{Rook Theory Interpretations}

\subsection{First $q$-Analogues}

In this section, we shall give combinatorial interpretations of the connection coefficients $r_{n, k}^{\mathcal{T}, \mathcal{S} \downarrow}(q), r_{n, k}^{\mathcal{T} \downarrow, \mathcal{S} \uparrow}(q), r_{n, k}^{\mathcal{T}, \mathcal{S} \uparrow}(q)$, and $r_{n, k}^{\mathcal{T} \downarrow, \mathcal{S} \downarrow}(q)$ for any two sequences of nonnegative integers $\mathcal{S}=\left(s_{1}, s_{2}, \ldots\right)$ and $\mathcal{T}=\left(t_{1}, t_{2}, \ldots\right)$. Recall that $S_{k}=\sum_{i=1}^{k} s_{i}$ is the $k^{\text {th }}$ partial sum of the $s_{i}{ }^{\prime}$ s and $T_{k}=\sum_{i=1}^{k} t_{i}$ is the $k^{t h}$ partial sum of the $t_{i}$ 's. Also, define $\mathcal{B}=\left\{T_{i}\right\}_{i=1}^{n}$ and $\mathcal{A}=\left\{s_{i}\right\}_{i=1}^{n}$.

Now consider equations (1.5), (1.6), (1.7), and (1.8). Each of these formulas are special cases of (2.4) for a particular general augmented board, namely $\mathcal{B}_{x}^{\mathcal{A}}$ for a fixed $n$, and particular choices of the sign functions. That is, if we want to produce a rising factorial $\langle x\rangle_{q} \uparrow_{n, \mathcal{T}}$ on the left-hand side of (2.4), we must have $\operatorname{Sgn}(i)=1$ for all $i$ and if we want to produce a falling factorial $\langle x\rangle_{q} \downarrow_{n, \mathcal{T}}$ on the left-hand side of (2.4), we must have $\operatorname{Sgn}(i)=-1$. Similarly, if we want to produce rising factorials $\langle x\rangle_{q} \uparrow_{k, \mathcal{S}}$ on the right-hand side of (2.4), we must have $\overline{\operatorname{Sgn}}(i)=1$ for all $i$ and if we want to produce falling factorials $\langle x\rangle_{q} \downarrow_{k, \mathcal{S}}$ on the right-hand side of (2.4), we must have $\overline{\operatorname{Sgn}}(i)=-1$. In this situation, the resulting augmented board that lies above the high bar is $\mathcal{B}^{\mathcal{A}}$ where $\mathcal{B}$ and $\mathcal{A}$ are defined for a fixed $n$ above. The $q$-weights $\mu_{q, \mathcal{B}^{\mathcal{A}}}^{\mathrm{Sgn}}(c)$ assigned to the cells in $\mathcal{B}^{\mathcal{A}}$ of course depend on the sign functions Sgn, $\overline{\operatorname{Sgn}}:[n] \rightarrow\{-1,1\}$. However the weights $\nu_{q, \mathcal{B}^{\mathcal{A}}}(c)=\left|\mu_{q, \mathcal{B}_{x}^{\mathcal{A}}}^{\mathrm{Sggn}}(c)\right|$ depend only on $\mathcal{B}^{\mathcal{A}}$. That is, in the $i^{\text {th }}$ column the $q$ weights of the cells in the base part of $\mathcal{B}^{\mathcal{A}}$ are $1, q, q^{2}, \ldots, q^{T_{i}-1}$, reading from bottom to top, and the $q$-weights of the cells in the augmented part are $1, q, q^{2}, \ldots, q^{S_{i}-1}$, reading from bottom to top. For example, if $n=5, \mathcal{T}=(0,2,2, \ldots)$ and $\mathcal{S}=(0,3,3, \ldots)$, then the weights on the board $\mathcal{B}^{\mathcal{A}}$ are pictured on the left of Figure 5 . If $\mathcal{T}=(0,3,3, \ldots)$ and $\mathcal{S}=(0,2,2, \ldots)$ then the weights on the board $\mathcal{B}^{\mathcal{A}}$ are pictured on the right of Figure 5 .

If $\mathbb{P}$ is a rook placement with rooks in cells $c_{1}, \ldots, c_{k}$, then we let

$$
\nu_{q, \mathcal{B}_{\mathcal{A}}}(\mathbb{P})=\prod_{i=1}^{k} \nu_{q, \mathcal{B}^{\mathcal{A}}}\left(c_{i}\right) .
$$

We now are in a position to give our combinatorial interpretations of $r_{n, k}^{\mathcal{T}, \mathcal{S} \downarrow}(q)$, $r_{n, k}^{\mathcal{T} \downarrow \mathcal{S} \uparrow}(q), r_{n, k}^{\mathcal{T}, \mathcal{S} \uparrow}(q)$, and $r_{n, k}^{\mathcal{T} \downarrow \mathcal{S} \downarrow}(q)$. We shall consider each case separately.

Case I: A combinatorial interpretation of $r_{n, k}^{\mathcal{T} \uparrow, \mathcal{S} \downarrow}(q)$.

If we let $\operatorname{Sgn}(i)=1$ and $\overline{\operatorname{Sgn}}(i)=-1$ for all $i$, then (2.4) becomes

$$
\langle x\rangle_{q} \uparrow_{n, \mathcal{T}}=\sum_{k=0}^{n} r_{n-k}\left(\mathcal{B}^{\mathcal{A}}, \operatorname{Sgn}, \overline{\operatorname{Sgn}}, q\right)\langle x\rangle_{q} \downarrow_{k, \mathcal{S}} .
$$

Comparing (1.5) and (3.2), we see that $r_{n, k}^{\mathcal{T}, \mathcal{S} \downarrow}(q)=r_{n-k}\left(\mathcal{B}^{\mathcal{A}}, \operatorname{Sgn}, \overline{\mathrm{Sgn}}, q\right)$ in the case where $\mathcal{B}=\left\{T_{1}, \ldots, T_{n}\right\}, \mathcal{A}=\left\{s_{1}, \ldots, s_{n}\right\}, \operatorname{Sgn}(i)=1$, and $\overline{\operatorname{Sgn}}(i)=-1$ for all $i$. Note 

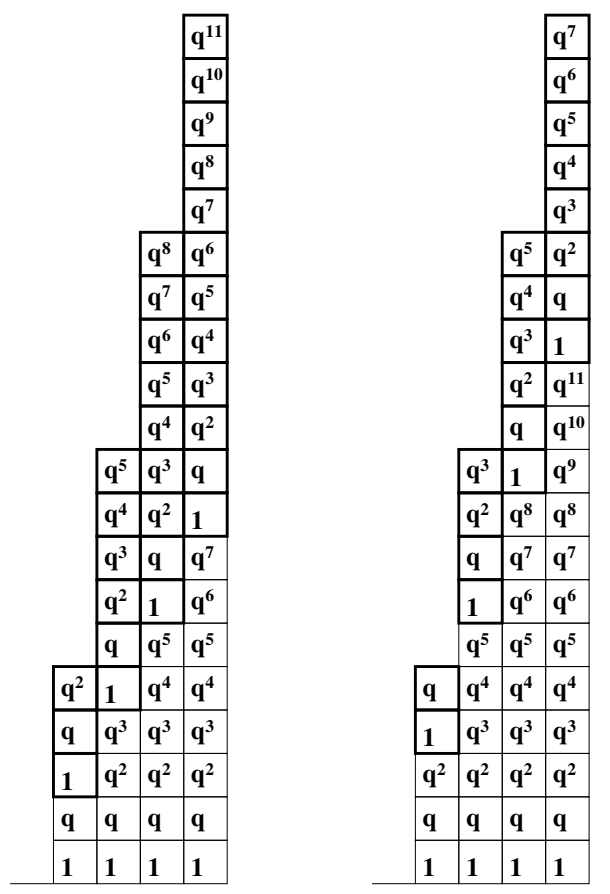

Figure 5: For $n=5$, the weights $\nu_{q, \mathcal{B}^{\mathcal{A}}}(c)$ when $\mathcal{T}=(0,2,2,2, \ldots), \mathcal{S}=(0,3,3,3, \ldots)$ and $\mathcal{T}=(0,3,3,3, \ldots), \mathcal{S}=(0,2,2,2, \ldots)$.

that in this case, the sign of each cell in $\mathcal{B}^{\mathcal{A}}$ is positive so that

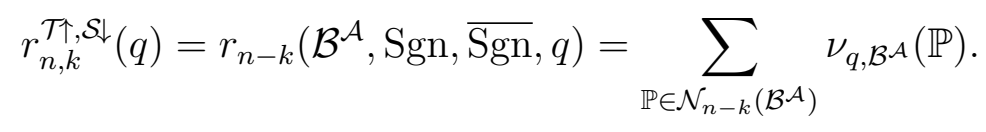

Case II: A combinatorial interpretation of $r_{n, k}^{\mathcal{T} \downarrow \mathcal{S} \uparrow}(q)$.

Now if we let $\operatorname{Sgn}(i)=-1$ and $\overline{\operatorname{Sgn}}(i)=1$ for all $i$, then (2.4) becomes

$$
\langle x\rangle_{q} \downarrow_{n, \mathcal{T}}=\sum_{k=0}^{n} r_{n-k}\left(\mathcal{B}^{\mathcal{A}}, \operatorname{Sgn}, \overline{\operatorname{Sgn}}, q\right)\langle x\rangle_{q} \uparrow_{k, \mathcal{S}} .
$$

Comparing (1.6) and (3.4), we see that $r_{n, k}^{\mathcal{T} \downarrow, \mathcal{S} \uparrow}(q)=r_{n-k}\left(\mathcal{B}^{\mathcal{A}}, \operatorname{Sgn}, \overline{\operatorname{Sgn}}, q\right)$ in the case where $\mathcal{B}=\left\{T_{1}, \ldots, T_{n}\right\}, \mathcal{A}=\left\{s_{1}, \ldots, s_{n}\right\}, \operatorname{Sgn}(i)=-1$, and $\overline{\operatorname{Sgn}}(i)=1$ for all $i$. Note that in this case, the sign of all cells in $\mathcal{B}^{\mathcal{A}}$ is -1 so that

$$
r_{n, k}^{\mathcal{T} \downarrow, \mathcal{S} \uparrow}(q)=r_{n-k}\left(\mathcal{B}^{\mathcal{A}}, \operatorname{Sgn}, \overline{\operatorname{Sgn}}, q\right)=(-1)^{n-k} \sum_{\mathbb{P} \in \mathcal{N}_{n-k}\left(\mathcal{B}^{\mathcal{A}}\right)} \nu_{q, \mathcal{B}^{\mathcal{A}}}(\mathbb{P}) .
$$

Case III: A combinatorial interpretation of $r_{n, k}^{\mathcal{T \uparrow} \mathcal{S} \uparrow}(q)$.

Next consider the case where $\operatorname{Sgn}(i)=1$ and $\overline{\operatorname{Sgn}}(i)=1$ for all $i$. Then (2.4) becomes

$$
\langle x\rangle_{q} \uparrow_{n, \mathcal{T}}=\sum_{k=0}^{n} r_{n-k}\left(\mathcal{B}^{\mathcal{A}}, \operatorname{Sgn}, \overline{\operatorname{Sgn}}, q\right)\langle x\rangle_{q} \uparrow_{k, \mathcal{S}} .
$$


Comparing (1.7) and (3.6), we see that $r_{n, k}^{\mathcal{T}, \mathcal{S} \uparrow}(q)=r_{n-k}\left(\mathcal{B}^{\mathcal{A}}, \operatorname{Sgn}, \overline{\operatorname{Sgn}}, q\right)$ in the case where $\mathcal{B}=\left\{T_{1}, \ldots, T_{n}\right\}, \mathcal{A}=\left\{s_{1}, \ldots, s_{n}\right\}, \operatorname{Sgn}(i)=1$, and $\overline{\operatorname{Sgn}}(i)=1$ for all $i$. Now in this case, the sign of the cells in the base part of $\mathcal{B}^{\mathcal{A}}$ is 1 and the sign of the cells in the augmented part of $\mathcal{B}^{\mathcal{A}}$ is -1 . Unlike the situation with $r_{n, k}^{\mathcal{T}, \mathcal{S} \downarrow}(q)$ and $r_{n, k}^{\mathcal{T \downarrow}, \mathcal{S} \uparrow}(q)$, there is cancelation among the weights of the rook placements in $\mathcal{N}_{n-k}\left(\mathcal{B}^{\mathcal{A}}\right)$. However in this case, we can construct a simple involution to eliminate many such cancelations.

Fix a placement $\mathbb{P} \in r_{n-k}\left(\mathcal{B}^{\mathcal{A}}, \operatorname{Sgn}, \overline{\operatorname{Sgn}}, q\right)$. Fix a column $i$ of $\mathcal{B}^{\mathcal{A}}$ and suppose that there are $f_{i}$ rooks to the left of column $i$ in $\mathbb{P}$. Then the number of cells in the base part of $\mathcal{B}^{\mathcal{A}}$ is $T_{i}$ and the number of uncanceled cells in the augmented part of $\mathcal{B}^{\mathcal{A}}$ is $S_{i-f_{i}}$. Now if $T_{i} \leq S_{i-f_{i}}$, then we shall refer to the base part of column $i$ as the primary part of column $i$ for $\mathbb{P}$ and the first $T_{i}$ cells of the augmented part of $\mathcal{B}^{\mathcal{A}}$ in column $i$, reading from bottom to top, as the mirror image of the primary part of column $i$ for $\mathbb{P}$. Similarly, if $T_{i}>S_{i-f_{i}}$, then we shall refer to first $S_{i-f_{i}}$ cells, reading from bottom to top, of the augmented part of column $i$ as the primary part of column $i$ for $\mathbb{P}$ and the first $S_{i-f_{i}}$ cells of the base part of $\mathcal{B}^{\mathcal{A}}$ in column $i$, reading from bottom to top, as the mirror image of the primary part of column $i$ for $\mathbb{P}$. Note that in each column $i$, the corresponding weights $\nu_{q, \mathcal{B}^{\mathcal{A}}}(c)$ of the cell in the $j^{\text {th }}$ row of the primary part of $\mathcal{B}^{\mathcal{A}}$, reading from bottom to top, and the cell in the $j^{\text {th }}$ row of the mirror image of the primary part of $\mathcal{B}^{\mathcal{A}}$, reading from bottom to top are the same, namely, $q^{j-1}$. This observation about these weights will be necessary to prove that our proposed involution is actually weight preserving.

For example, if $\mathcal{T}=(1,3,1,3,1,3, \ldots)$ and $\mathcal{S}=(2,2,2,2, \ldots)$, then for $n=5$, we picture the board $\mathcal{B}^{\mathcal{A}}$ in Figure 6 on the left. Moreover, in Figure 6, we inserted the corresponding $q$-weights, $\nu_{q, \mathcal{B}^{\mathcal{A}}}(\mathbb{P})=\left|\mu_{q, \mathcal{B}^{\mathcal{A}}}^{\mathrm{Sgn}}, \overline{\mathrm{Sg}}(\mathbb{P})\right|$, of each cell. On the right, we have pictured a rook placement $\mathbb{P}$ and we have indicated the primary part of each column for $\mathbb{P}$ by the darkly shaded cells and mirror image of the primary part for $\mathbb{P}$ by the lightly shaded cells. The cells which are blank are the cells that lie neither in the primary part nor in the mirror image of the primary part in their column.

We define $I: \mathcal{N}_{n-k}\left(\mathcal{B}^{\mathcal{A}}\right) \rightarrow \mathcal{N}_{n-k}\left(\mathcal{B}^{\mathcal{A}}\right)$ as follows. If a placement $\mathbb{P} \in \mathcal{N}_{n-k}\left(\mathcal{B}^{\mathcal{A}}\right)$ has a rook that lies either in the primary part or the mirror image for $\mathbb{P}$ of the primary part of some column $j$ in $\mathcal{B}^{\mathcal{A}}$, then let $i$ be the leftmost column that contains such a rook. Then the involution $I$ is very simple, namely, if $r$ is a rook in column $i$ and $r$ is in the $j^{\text {th }}$ row of the primary part for $\mathbb{P}$ in column $i$, reading from bottom to top, then $I(\mathbb{P})$ is defined to be the rook placement that results by moving $r$ to the $j^{\text {th }}$ row of the mirror image of the primary part for $\mathbb{P}$, reading from bottom to top. Vice versa, if $r$ is in the $j^{\text {th }}$ row of the mirror image of the primary part for $\mathbb{P}$ in column $i$, reading from bottom to top, then $I(\mathbb{P})$ is defined to be the rook placement that results by moving $r$ to the $j^{\text {th }}$ row of the primary part for $\mathbb{P}$ in column $i$, reading from bottom to top. By our observation about the weights of these cells being the same, it follows that $\mu_{q, \mathcal{B}^{\mathcal{A}}}^{\mathrm{Sgn}}(\mathbb{P})=-\mu_{q, \mathcal{B}^{\mathcal{A}}}^{\mathrm{Sggn}}(I(\mathbb{P}))$. Finally if $\mathbb{P}$ does not have a rook that lies in the primary part for $\mathbb{P}$ or in the mirror image of primary part for $\mathbb{P}$ in any column, then we set $I(\mathbb{P})=\mathbb{P}$. For example, in the case where $\mathcal{T}=(1,3,1,3, \ldots)$ and $\mathcal{S}=(2,2,2,2, \ldots)$, Figure 7 demonstrates the involution $I(\mathbb{P})$ for $\mathbb{P} \in \mathcal{N}_{3}\left(\mathcal{B}^{\mathcal{A}}\right)$ when $n=5$. 

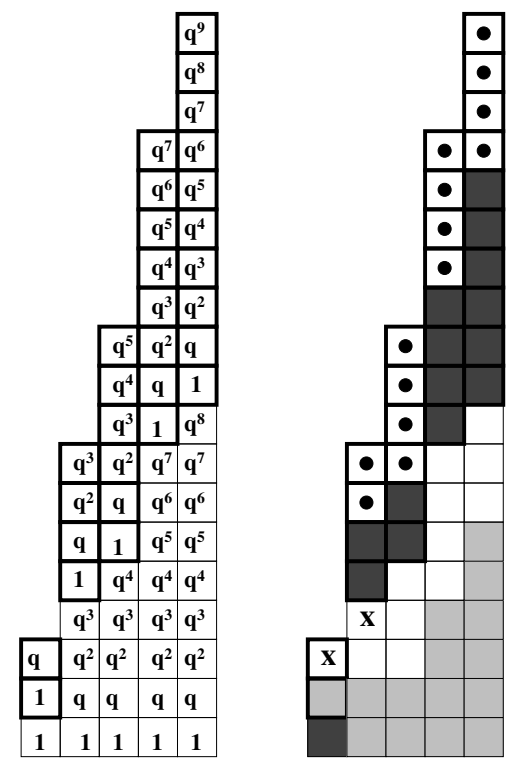

Figure 6: The board $\mathcal{B}^{\mathcal{A}}$ when $n=5, \mathcal{T}=(1,3,1,3, \ldots)$ and $\mathcal{S}=(2,2,2,2, \ldots)$.

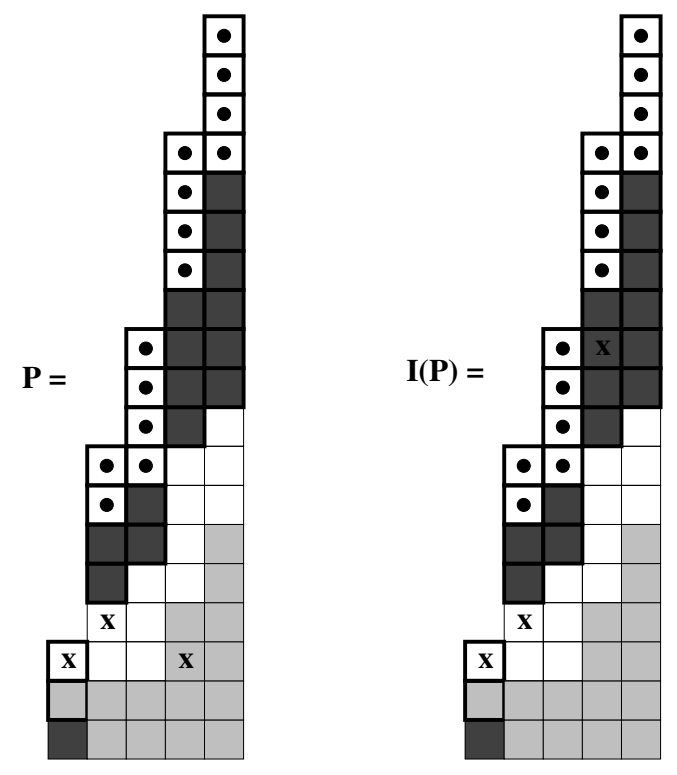

Figure 7: An example of the involution $I$ for $\mathcal{B}^{\mathcal{A}}$ where $n=5, \mathcal{T}=(1,3,1,3, \ldots)$ and $\mathcal{S}=(2,2,2,2, \ldots)$. 
We see from our definitions that $I^{2}$ is the identity. Thus, $I$ is a sign-reversing, weight-preserving involution, so that if we let $\mathcal{F} N_{n-k}\left(\mathcal{B}^{\mathcal{A}}\right)$ denote the set of all $\mathbb{P} \in$ $\mathcal{N}_{n-k}\left(\mathcal{B}^{\mathcal{A}}\right)$ such that $I(\mathbb{P})=\mathbb{P}$, then $I$ shows that

$$
r_{n, k}^{\mathcal{T} \uparrow, \mathcal{S} \uparrow}(q)=\sum_{\mathbb{P} \in \mathcal{N}_{n-k}\left(\mathcal{B}^{\mathcal{A}}\right)} \mu_{q, \mathcal{B}^{\mathcal{A}}}^{\mathrm{Sgn}, \overline{, g n}}(\mathbb{P})=\sum_{\mathbb{P} \in \mathcal{F} N_{n-k}\left(\mathcal{B}^{\mathcal{A}}\right)} \mu_{q,\left(\mathcal{B}^{\mathcal{A}}\right)}^{\mathrm{Sgn}, \overline{P g}}(\mathbb{P}) .
$$

In some special cases, the involution $I$ has eliminated all the possible cancelations. For example, consider the following two cases.

Case III.a $T_{i} \geq S_{i}$ for all $i=1, \ldots, n$.

In this case, we see that all the uncanceled cells that lie in the augmented part of the board $\mathcal{B}^{\mathcal{A}}$ for some rook placement $\mathbb{P}$ in $\mathcal{B}^{\mathcal{A}}$ must lie either in the primary part or the mirror images of the primary part for $\mathbb{P}$. This means that if $I(\mathbb{P})=\mathbb{P}$, then all the rooks in $\mathbb{P}$ must lie in the base part of $\mathcal{B}^{\mathcal{A}}$. Since the signs of each cell in the base part of $\mathcal{B}^{\mathcal{A}}$ is positive in this case, it follows that

$$
r_{n, k}^{\mathcal{T} \uparrow, \mathcal{S} \uparrow}(q)=\sum_{\mathbb{P} \in \mathcal{F} N_{n-k}\left(\mathcal{B}^{\mathcal{A}}\right)} \nu_{q, \mathcal{B}^{\mathcal{A}}}(\mathbb{P}) .
$$

Case III.b $T_{i} \leq S_{1}$ for all $i=1, \ldots, n$.

In this case, we see that for any rook placement $\mathbb{P}$, there will be at least $S_{1}$ uncanceled cells in $\mathcal{B}^{\mathcal{A}}$. Hence it will be that case that the primary part of each column $i$ is the base part of column $i$. This means that if $I(\mathbb{P})=\mathbb{P}$, then all the rooks in $\mathbb{P}$ must lie the augmented part of $\mathcal{B}^{\mathcal{A}}$. Since the signs of each cell in the augmented part of $\mathcal{B}^{\mathcal{A}}$ is negative in this case, it follows that

$$
r_{n, k}^{\mathcal{T} \uparrow, \mathcal{S} \uparrow}(q)=(-1)^{n-k} \sum_{\mathbb{P} \in \mathcal{F} N_{n-k}\left(\mathcal{B}^{\mathcal{A}}\right)} \nu_{q, \mathcal{B}^{\mathcal{A}}}(\mathbb{P}) .
$$

However, in general, elements of $\mathcal{F} N_{n-k}\left(\mathcal{B}^{\mathcal{A}}\right)$ can still have rooks in both the base part and the augmented part of $\mathcal{B}^{\mathcal{A}}$. For example, if $n=5, \mathcal{T}=(1,3,1,3,1,3, \ldots)$, $\mathcal{S}=(2,2,2,2, \ldots)$, and we consider the rook placement $\mathbb{P}$ pictured on the right in Figure 6, then it is easy to see that $\mathbb{P} \in \mathcal{F} N_{2}\left(\mathcal{B}^{\mathcal{A}}\right)$ has rooks in the both the base part and the augmented part of $\mathcal{B}^{\mathcal{A}}$.

In fact, in general it is not the case that the terms in $r_{n, k}^{\mathcal{T \uparrow} \mathcal{S} \uparrow}(q)$ have the same sign. For example, if $n=3, \mathcal{T}=(1,3,3,3, \ldots)$ and $\mathcal{S}=(2,2,2,2, \ldots)$, then in Figure 8, we have pictured the primary and the mirror image of the primary parts of the columns depending whether one has no rook in column 1, one rook in column 1 that does not lie in the either the primary part or the mirror image of the primary part column 1 , and two rooks in columns 1 and 2 where no rook lies in the either the primary parts or the mirror image of the primary parts columns 1 and 2. Again, in each column, we have used the darkly shaded cells to represent the primary part of the column for 

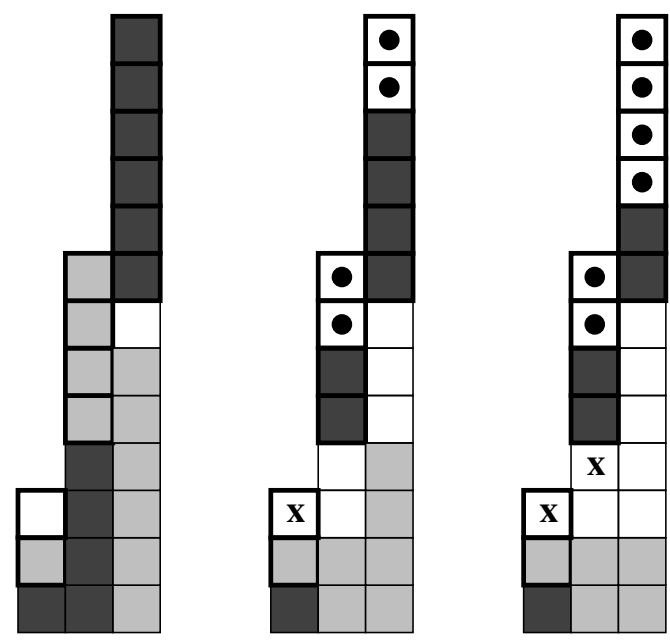

Figure 8: Placements in $\mathcal{F} N_{1}\left(\mathcal{B}^{\mathcal{A}}\right)$ where $n=3, \mathcal{T}=(1,3,3,3, \ldots)$ and $\mathcal{S}=$ $(2,2,2,2, \ldots)$.

$\mathbb{P}$ and the lightly shaded cells to represent the mirror image of the primary part for $\mathbb{P}$. The connection coefficient $r_{3,3}^{\mathcal{T}, \mathcal{S}}(q)=1$ since it corresponds to the placement of no rooks in $\mathcal{B}^{\mathcal{A}}$. Next, the connection coefficient $r_{3,2}^{\mathcal{T}, \mathcal{S} \uparrow}(q)$ corresponds to the placement of one rook in $\mathcal{B}^{\mathcal{A}}$. If we do not place a rook in either the primary part or the mirror image of the primary part, then either the rook must go in cell $(3,1)$ so that its weight is $-q$ or, if there no rook in column 1, then we cannot place a rook in column 2 so that the only other possibility is to place the rook in cell $(7,3)$ which has weight $q^{6}$. Thus $r_{3,2}^{\mathcal{T} \uparrow, \mathcal{S} \uparrow}(q)=q^{6}-q$. The connection coefficient $r_{3,1}^{\mathcal{T} \uparrow \mathcal{S} \uparrow}(q)$ corresponds to the placement of two rooks in $\mathcal{B}^{\mathcal{A}}$. We cannot place the two rooks in columns 2 and 3 because if we do not place a rook in column 1 , there are no squares in column 2 which are not in either the primary or the mirror image of the primary part. It then is easy to see that from the middle diagram in Figure 8 that if we place a rook in the only possible cell in column 1 , namely cell $(3,1)$ which has weight $-q$, then we can either place the second rook in cells $(3,2),(4,2),(5,3),(6,3)$, or $(7,3)$ which have weights $q^{2}, q^{3}, q^{4}, q^{5}, q^{6}$, respectively, so that $r_{3,1}^{\mathcal{T}, \mathcal{S} \uparrow}(q)=-q\left(q^{2}+q^{3}+q^{4}+q^{5}+q^{6}\right)$. Finally, it is easy to see from the third diagram in Figure 8 that if we place a rook in the only possible cell in column 1, namely cell $(3,1)$ which has weight $-q$, then we can place the second rook in either cell $(3,2)$ or $(4,2)$ which have weights $q^{2}$ and $q^{3}$, respectively, and the third rook in either cells $(3,3)$, $(4,3),(5,3),(6,3)$, or $(7,3)$ which have weights $q^{2}, q^{3}, q^{4}, q^{5}$, and $q^{6}$, respectively, so that $r_{3,0}^{\mathcal{T}, \mathcal{S} \uparrow}(q)=-q\left(q^{2}+q^{3}\right)\left(q^{2}+q^{3}+q^{4}+q^{5}+q^{6}\right)$.

Case IV: A combinatorial interpretation of $r_{n, k}^{\mathcal{T \downarrow}, \mathcal{S} \downarrow}(q)$.

Next consider the case where $\operatorname{Sgn}(i)=-1$ and $\overline{\operatorname{Sgn}}(i)=-1$ for all $i$. Then (2.4) becomes 


$$
\langle x\rangle_{q} \downarrow_{n, \mathcal{T}}=\sum_{k=0}^{n} r_{n-k}\left(\mathcal{B}^{\mathcal{A}}, \operatorname{Sgn}, \overline{\operatorname{Sgn}}, q\right)\langle x\rangle_{q} \downarrow_{k, \mathcal{S}} .
$$

Comparing (1.8) and (3.10), we see that $r_{n, k}^{\mathcal{T \downarrow}, \mathcal{S} \downarrow}(q)=r_{n-k}\left(\mathcal{B}^{\mathcal{A}}, \operatorname{Sgn}, \overline{\operatorname{Sgn}}, q\right)$ in the case where $\mathcal{B}=\left\{T_{1}, \ldots, T_{n}\right\}, \mathcal{A}=\left\{s_{1}, \ldots, s_{n}\right\}, \operatorname{Sgn}(i)=-1$, and $\overline{\operatorname{Sgn}}(i)=-1$ for all $i$. Now in this case, the sign of the cells in the base part $\mathcal{B}^{\mathcal{A}}$ is -1 and the sign of the cells in augmented part of $\mathcal{B}^{\mathcal{A}}$ is 1 . Hence, the involution $I$ defined in Case III is still a sign-reversing, weight-preserving involution. Thus $I$ shows that

$$
r_{n, k}^{\mathcal{T} \downarrow, \mathcal{S} \downarrow}(q)=\sum_{\mathbb{P} \in \mathcal{N}_{n-k}\left(\mathcal{B}^{\mathcal{A}}\right)} \mu_{q, \mathcal{B}^{\mathcal{A}}}^{\mathrm{Sggn}}(\mathbb{P})=\sum_{\mathbb{P} \in \mathcal{F} N_{n-k}\left(\mathcal{B}^{\mathcal{A}}\right)} \mu_{q, \mathcal{B}^{\mathcal{A}}}^{\mathrm{Sgn}} \overline{\overline{\mathrm{Sgn}}}(\mathbb{P}) .
$$

Again, in some special cases, the involution $I$ has eliminated all the possible cancelations. For example, we obtain the following two cases which are similar to CaseIII.a and CaseIII.b.

Case IV.a $T_{i} \geq S_{i}$ for all $i=1, \ldots, n$.

In this case,

$$
r_{n, k}^{\mathcal{T} \downarrow, \mathcal{S} \downarrow}(q)=(-1)^{n-k} \sum_{\mathbb{P} \in \mathcal{F} N_{n-k}\left(\mathcal{B}^{\mathcal{A}}\right)} \nu_{q, \mathcal{B}^{\mathcal{A}}}(\mathbb{P}) .
$$

Case IV.b $T_{i} \leq S_{1}$ for all $i=1, \ldots, n$.

In this case,

$$
r_{n, k}^{\mathcal{T} \downarrow \mathcal{S} \downarrow}(q)=\sum_{\mathbb{P} \in \mathcal{F} N_{n-k}\left(\mathcal{B}^{\mathcal{A}}\right)} \nu_{q, \mathcal{B}^{\mathcal{A}}}(\mathbb{P})
$$

\subsection{Modified $q$-Analogues}

We note that in our $q$-analogue of the general product formula (2.4), we have taken the $q$-analogue of $(x+a)$ to be $[x]_{q}+[a]_{q}$ and the $q$-analogue of $(x-a)$ to be $[x]_{q}-[a]_{q}$ if $x$ and $a$ are nonnegative integers. Miceli and Remmel, see section 4.1 in [10], showed that one could modify the $q$-weights of the cells in $\mathcal{B}_{x}^{\mathcal{A}}$ in order to produce a $q$-analogue of the general product formula where the $q$-analogue of $(x+a)$ is $[x+a]_{q}$ and the $q$-analogue of $(x-a)$ is $[x-a]_{q}$. The basic idea of Miceli and Remmel was to modify (2.4) by using the following simple identities which hold when $x$ and $a$ are nonnegative integers with $x \geq a$ :

$$
[x]_{q}-[a]_{q}=q^{a}[x-a]_{q}
$$


and

$$
[x]_{q}+q^{x}[a]_{q}=[x+a]_{q} .
$$

Throughout this section, we shall fix two sequences of nonnegative integers $\mathcal{S}=$ $\left(s_{1}, s_{2}, \ldots\right)$ and $\mathcal{T}=\left(t_{1}, t_{2}, \ldots\right)$ and again we set $S_{k}=\sum_{i=1}^{k} s_{i}$ and $T_{k}=\sum_{i=1}^{k} t_{i}$ for $i \geq 1$. We then let $\mathcal{B}=\left(T_{1}, \ldots, T_{n}\right)$ and $\mathcal{A}=\left(s_{1}, \ldots, s_{n}\right)$. For our purposes, we need only recall how Miceli and Remmel modified the $q$-rook numbers to prove such $q$-analogues of our general product formula in the case where Sgn and $\overline{\operatorname{Sgn}}$ are each constant on $[n]$. This is simply because only in this situation does (2.4) specialize to an expression relating generalized rising and falling factorial bases. Thus, there are only four cases to consider. As this is thoroughly explained in [10], we will only demonstrate how to modify the weights in one of the cases and will state the result for the remaining three. We will consider the case where $\operatorname{Sgn}(i)=\overline{\operatorname{Sgn}}(i)=1$ (this is actually Case III in [10]). Case I: $\operatorname{Sgn}(i)=\overline{\operatorname{Sgn}}(i)=1$.

In this case, (2.4) becomes

$$
\prod_{i=1}^{n}\left([x]_{q}+\left[T_{i}\right]_{q}\right)=\sum_{k=0}^{n} r_{n-k}\left(\mathcal{B}^{\mathcal{A}}, \operatorname{Sgn}, \overline{\operatorname{Sgn}}, q\right) \prod_{r=1}^{k}\left([x]_{q}+\left[S_{r}\right]_{q}\right) .
$$

We would like to replace the factors $\left([x]_{q}+\left[T_{i}\right]_{q}\right)$ by $\left([x]_{q}+q^{x}\left[T_{i}\right]_{q}\right)=\left[x+T_{i}\right]_{q}$ and the factors $\left([x]_{q}+\left[S_{r}\right]_{q}\right)$ by $\left([x]_{q}+q^{x}\left[S_{r}\right]_{q}\right)=\left[x+S_{r}\right]_{q}$. To do this, Miceli and Remmel observed from their proof of Theorem 2.1 that we need only weight each cell in the base part of $\mathcal{B}_{x}^{\mathcal{A}}$ and each cell in both the lower and the upper augmented parts of $\mathcal{B}_{x}^{\mathcal{A}}$ with an extra factor of $q^{x}$. Thus, for any $\mathbb{P} \in \mathcal{N}_{k}\left(\mathcal{B}^{\mathcal{A}}\right)$, we set

$$
\overline{\bar{\mu}}_{q, \mathcal{B}^{\mathcal{A}}}^{\mathrm{Sggn}}(\mathbb{P})=q^{x(\operatorname{Base}(\mathbb{P})+\operatorname{UAug}(\mathbb{P})+\operatorname{LAug}(\mathbb{P}))} \mu_{q, \mathcal{B}^{\mathcal{A}}}^{\operatorname{Sgn}, \overline{\operatorname{Sgn}}}(\mathbb{P}),
$$

where $\operatorname{Base}(\mathbb{P}), \operatorname{UAug}(\mathbb{P})$, and $\operatorname{LAug}(\mathbb{P})$ are the number of rooks of $\mathbb{P}$ which lie in the base part, the upper augmented part, and the lower augmented part of the board $\mathcal{B}^{\mathcal{A}}$, respectively. Define

$$
\overline{\bar{r}}_{n-k}\left(\mathcal{B}^{\mathcal{A}}, q\right)=\sum_{\mathbb{P} \in \mathcal{N}_{n-k}\left(\mathcal{B}^{\mathcal{A}}\right)} \overline{\bar{\mu}}_{q, \mathcal{B}^{\mathcal{A}}}^{\mathrm{Sgn}, \overline{\mathrm{Sgn}}}(\mathbb{P}) .
$$

Then Miceli and Remmel (equation 4.19 in [10]) proved that

$$
\prod_{i=1}^{n}\left(\left[x+T_{i}\right]_{q}\right)=\sum_{k=0}^{n} \overline{\bar{r}}_{n-k}^{\mathcal{A}}\left(\mathcal{B}^{\mathcal{A}}, q\right)\left(\left[x+S_{1}\right]_{q}\right)\left(\left[x+S_{2}\right]_{q}\right) \cdots\left(\left[x+S_{k}\right]_{q}\right),
$$

giving that (3.19) becomes

$$
[x]_{q} \uparrow_{\mathcal{T}, n}=\sum_{k=1}^{n} \overline{\bar{r}}_{n-k}^{\mathcal{A}}\left(\mathcal{B}^{\mathcal{A}}, q\right)[x]_{q} \uparrow_{\mathcal{S}, k} .
$$


Comparing (1.11) and (3.20), it follows that

$$
\tilde{r}_{n, k}^{\mathcal{T \uparrow}, \mathcal{S} \uparrow}(q)=\overline{\bar{r}}_{n-k}^{\mathcal{A}}\left(\mathcal{B}^{\mathcal{A}}, q\right)=q^{(n-k) x} r_{n, k}^{\mathcal{T}, \mathcal{S} \uparrow}(q) .
$$

Case II: $\operatorname{Sgn}(i)=\overline{\operatorname{Sgn}}(i)=-1$.

In this case, we obtain that

$$
\tilde{r}_{n-k}^{\mathcal{T} \downarrow, \mathcal{S} \downarrow}(q)=q^{\left(S_{1}+S_{2}+\cdots+S_{k}\right)-\left(T_{1}+\cdots+T_{n}\right)} r_{n-k}^{\mathcal{T} \downarrow, \mathcal{S} \downarrow}(q) .
$$

Case III: $\operatorname{Sgn}(i)=+1, \overline{\operatorname{Sgn}}(i)=-1$.

In this case, for any $\mathbb{P} \in \mathcal{N}_{k}\left(\mathcal{B}^{\mathcal{A}}\right)$, set

$$
\hat{\mu}_{q, \mathcal{B}^{\mathcal{A}}}^{\mathrm{Sgn}}(\overline{\mathbb{S g n}})=q^{x(\operatorname{Base}(\mathbb{P}))} \mu_{q, \mathcal{B}^{\mathcal{A}}}^{\mathrm{Sgn}} \overline{\widehat{\operatorname{Sgn}}}(\mathbb{P}),
$$

and

$$
\hat{r}_{n-k}\left(\mathcal{B}^{\mathcal{A}}, q\right)=\sum_{\mathbb{P} \in \mathcal{N}_{n-k}\left(\mathcal{B}^{\mathcal{A}}\right)} q^{S_{1}+\cdots+S_{k}} \hat{\mu}_{q, \mathcal{B}^{\mathcal{A}}}^{\mathrm{Sggn}}(\mathbb{P}) .
$$

We then obtain that if $x \geq S_{n}$,

$$
\prod_{i=1}^{n}\left(\left[x+T_{i}\right]_{q}\right)=\sum_{k=0}^{n} \hat{r}_{n-k}\left(\mathcal{B}^{\mathcal{A}}, q\right)\left(\left[x-S_{1}\right]_{q}\right)\left(\left[x-S_{2}\right]_{q}\right) \cdots\left(\left[x-S_{k}\right]_{q}\right) .
$$

Therefore,

$$
\tilde{r}_{n, k}^{\mathcal{T}, \mathcal{S} \downarrow}(q)=\hat{r}_{n-k}\left(\mathcal{B}^{\mathcal{A}}, q\right)
$$

Case IV: $\operatorname{Sgn}(i)=-1, \overline{\operatorname{Sgn}}(i)=+1$.

In this case, for any $\mathbb{P} \in \mathcal{N}_{k}\left(\mathcal{B}^{\mathcal{A}}\right)$, set

$$
\hat{\hat{\mu}}_{q, \mathcal{B}^{\mathcal{A}}}^{\mathrm{Sgg}} \overline{\operatorname{Sgn}}(\mathbb{P})=q^{x(\operatorname{UAug}(\mathbb{P})+\operatorname{LAug}(\mathbb{P}))} \mu_{q, \mathcal{B} \mathcal{A}}^{\mathrm{Sgn}, \overline{\operatorname{Sgn}}}(\mathbb{P}),
$$

and

$$
\hat{\hat{r}}_{n-k}\left(\mathcal{B}^{\mathcal{A}}, q\right)=\sum_{\mathbb{P} \in \mathcal{N}_{n-k}\left(\mathcal{B}^{\mathcal{A}}\right)} q^{S_{1}+\cdots+S_{k}} \hat{\hat{\mu}}_{q, \mathcal{B}^{\mathcal{A}}}^{\mathrm{Sggn}}(\mathbb{P}) .
$$

We then obtain that if $x \geq S_{n}$,

$$
\prod_{i=1}^{n}\left(\left[x+T_{i}\right]_{q}\right)=\sum_{k=0}^{n} \hat{\hat{r}}_{n-k}\left(\mathcal{B}^{\mathcal{A}}, q\right)\left(\left[x-S_{1}\right]_{q}\right)\left(\left[x-S_{2}\right]_{q}\right) \cdots\left(\left[x-S_{k}\right]_{q}\right) .
$$


Therefore,

$$
\tilde{r}_{n, k}^{\mathcal{T}, \mathcal{S} \uparrow}(q)=\hat{\hat{r}}_{n-k}\left(\mathcal{B}^{\mathcal{A}}, q\right) .
$$

It is worth noting that in Case III, (2.4) becomes

$$
\prod_{i=1}^{n}\left([x]_{q}+\left[T_{i}\right]_{q}\right)=\sum_{k=0}^{n} r_{n-k}\left(\mathcal{B}^{\mathcal{A}}, \operatorname{Sgn}, \overline{\operatorname{Sgn}}, q\right) \prod_{r=1}^{k}\left([x]_{q}-\left[S_{r}\right]_{q}\right) .
$$

This in turn gives

$$
[x]_{q} \uparrow_{\mathcal{T}, n}=\sum_{k=1}^{n} \hat{r}_{n-k}\left(\mathcal{B}^{\mathcal{A}}, q\right)[x]_{q} \downarrow_{\mathcal{S}, k} .
$$

In the special case where $\mathcal{T}=\mathcal{S}=(0,1, \ldots, 1)$, (3.32) becomes

$$
\prod_{i=1}^{n}[x+i-1]_{q}=\sum_{k=1}^{n} \tilde{r}_{n, k}^{\uparrow \uparrow} \mathcal{S} \downarrow
$$

and if we replace $[x]_{q}$ by $x$ in (3.31), then we obtain that

$$
\prod_{i=1}^{n}\left(x+[i-1]_{q}\right)=\sum_{k=0}^{n} r_{n, k}^{\mathcal{T \uparrow}, \mathcal{S \downarrow}}(q) x\left(x-[1]_{q}\right)\left(x-[2]_{q}\right) \cdots\left(x-[k-1]_{q}\right) .
$$

These equations are two different $q$-analogues of the equation that define the Lah numbers $L_{n, k}$. It follows that $\tilde{r}_{n, k}^{\mathcal{T}, \mathcal{S} \downarrow}(q)=L_{n, k}(q)$ where $L_{n, k}(q)$, is the $q$-analog of the Lah number $L_{n, k}$ defined by Garsia and Remmel [3] who interpreted $L_{n, k}(q)$ a $q$-counting configurations of balls and tubes. Similarly, $r_{n, k}^{\mathcal{T}, \mathcal{S} \downarrow}(q)=L_{q}(n, k)$ where $L_{q}(n, k)$, is the $q$-analog of the Lah number $L_{n, k}$ defined by Lindsay, Mansour, and Shattuck [6], who also interpreted $L_{q}(n, k)$ as $q$-counting configurations of balls and tubes, but in a different way from Garsia and Remmel.

Remark. It is possible to use the ideas of Miceli and Remmel to find combinatorial interpretations of the connection coefficients between more general rising and falling factorial bases of the form $\left(x \pm \overline{S_{1}}\right)\left(x \pm \overline{S_{2}}\right) \cdots\left(x \pm \overline{S_{k}}\right)$ where $\mathcal{S}=\left(s_{1}, s_{2}, \ldots\right)$ is any sequence of nonnegative integers, $\operatorname{Sgn}: \mathbb{N}_{0} \rightarrow\{-1,1\}$, and $\overline{S_{k}}=\sum_{i=1}^{k} \operatorname{Sgn}(i) s_{i}$. Similarly, Miceli and Remmel indicated how to find $p, q$-analogues with their theory and, hence, we could find $p, q$-analogues of the results of this paper. In both cases, the combinatorial interpretations are necessarily more complicated than the ones presented in this paper and one can no longer give the type of uniform proofs that characterize our results here. 


\section{Connections with permutations and set partitions}

Recall that for any positive integer $n,[n]:=\{1,2, \ldots, n\}$. We note that in the special case where $\mathcal{T}=(0,0,0,0 \ldots)$ and $\mathcal{S}=(0,1,1,1, \ldots),(1.1)$ becomes

$$
x^{n}=\sum_{k=1}^{n} r_{n, k}^{\mathcal{T} \uparrow \mathcal{S} \downarrow}(x) \downarrow_{k},
$$

where $(x) \downarrow_{k}=x(x-1) \cdots(x-k+1)$ so that $r_{n, k}^{\mathcal{T} \uparrow, \mathcal{S} \downarrow}$ equals the classical Stirling number of the second kind, $S_{n, k}$, which counts the number of unordered set partitions of $[n]$ into $k$ nonempty parts. Similarly, (1.2) yields

$$
(x) \downarrow_{n}=\sum_{k=1}^{n} r_{n, k}^{\mathcal{S} \downarrow, \mathcal{T} \uparrow} x^{k},
$$

so that $r_{n, k}^{\mathcal{S} \downarrow, \mathcal{T} \uparrow}$ equals the classical signed Stirling number of the first kind, $s_{n, k}$, which is equal to $(-1)^{n-k}$ times the number of permutations of $[n]$ with $k$ cycles. Thus, it is natural to ask whether we can give combinatorial interpretations of $r_{n, k}^{\mathcal{T} \uparrow, \mathcal{S} \downarrow}(q), r_{n, k}^{\mathcal{T} \downarrow, \mathcal{S} \uparrow}(q)$, $r_{n, k}^{\mathcal{T} \uparrow, \mathcal{S} \uparrow}(q)$, and $r_{n, k}^{\mathcal{T} \downarrow, \mathcal{S} \uparrow}(q)$ in terms of permutations and set partitions.

As in the previous section, we shall fix finite sequences $\mathcal{T}=\left(t_{1}, \ldots, t_{n}\right)$ and $\mathcal{S}=$ $\left(s_{1}, \ldots, s_{n}\right)$ from $\mathbb{N}_{0}^{n}$ and consider the augmented board $\mathcal{B}^{\mathcal{A}}$ where $\mathcal{B}=\left(T_{1}, \ldots, T_{n}\right)$, $T_{k}=\sum_{i=1}^{k} t_{i}$ for $k=1, \ldots, n$, and $\mathcal{A}=\left(s_{1}, \ldots, s_{n}\right)$. The main goal of this section is to show that we can give combinatorial interpretations of $r_{n, k}^{\mathcal{T} \uparrow, \mathcal{S} \downarrow}(q), r_{n, k}^{\mathcal{T} \downarrow, \mathcal{S} \uparrow}(q), r_{n, k}^{\mathcal{T \uparrow}, \mathcal{S} \uparrow}(q)$, and $r_{n, k}^{\mathcal{T} \downarrow, \mathcal{S} \downarrow}(q)$ in terms of $q$-counting certain pairs $(\sigma, \pi)$, where $\sigma$ is colored permutation and $\pi$ is a colored set partition of $[n]$. Here we will use the colors $0,1,2, \ldots$ and indicate that a number $i$ is colored with color $j$ by writing $i_{j}$. In such a situation, we shall talk about the absolute value of $i_{j}$ as just being the number $i$ and write $\left|i_{j}\right|=i$. Then a colored permutation $\sigma=\sigma_{1} \ldots \sigma_{n}$ of $[n]$ is a sequence of colored numbers so that $|\sigma|=\left|\sigma_{1}\right| \ldots\left|\sigma_{n}\right|$ is a permutation in the symmetric group $S_{n}$. Similarly a colored set partition of $[n]$ is a set partition of colored numbers such that if we replace each colored number by its absolute value, then we obtain a set partition of $[n]$. For example, $\sigma=2_{1} 3_{2} 4_{1} 5_{0} \quad 7_{1} \quad 6_{2} 1_{2}$ is a colored permutation of [7] such that $|\sigma|=2345761$ and $\pi=\left\langle\left\{1_{0}, 3_{3}, 4_{1}\right\},\left\{2_{1}, 5_{2}\right\},\left\{6_{2}, 7_{1}\right\}\right\rangle$ is a colored set partition of [7] such that $|\pi|=\langle\{1,3,4\},\{2,5\},\{6,7\}\rangle$.

We let $C P e r m_{n, k}$ denote the set of colored permutations $\sigma$ of $[n]$ such that $|\sigma|$ is a permutation with $k$ cycles. We shall always order the cycles of $|\sigma|$ so that the smallest element in each cycle is on the left and the cycles are ordered by increasing smallest elements. We let $S M(\sigma)$ denote the set of smallest elements in the cycles of $|\sigma|$. For example, if $\sigma=\left(1_{1}, 4_{2}, 3_{1}\right)\left(2_{3}, 7_{1}, 5_{3}\right)\left(6_{2}\right)$, then $S M(\sigma)=\{1,2,6\}$. We let CSPtn $n_{n, k}$ denote the set of colored set partitions $\pi$ of $[n]$ such that $|\pi|$ is a set partition with $k$ nonempty parts. We shall always order the parts of $|\pi|$ by increasing smallest elements. We let $S M(\pi)$ denote the set of smallest elements in the parts of $|\pi|$. For example, if $\pi=\left\langle\left\{1_{1}, 4_{2}, 5_{1}\right\},\left\{2_{3}, 3_{1}, 6_{3}\right\},\left\{7_{2}\right\}\right\rangle$, then $S M(\pi)=\{1,2,7\}$. 
Next we give a key definition which will be used to map placements in $\mathcal{N}_{n-k}\left(\mathcal{B}^{\mathcal{A}}\right)$ onto certain pairs $(\sigma, \pi) \in C \operatorname{Perm}_{n, k} \times C S P t n_{n, k} \cup C \operatorname{Perm}_{n, k+1} \times C S P t n_{n, k+1}$.

Definition 1. 1. Given an element $\sigma \in C P e r m_{n, k}$, suppose that $\tau \in C P e r m_{n+1, k}$ is the result of inserting the colored number $n+1_{j}$ immediately after some colored number $i_{s}$ in $\sigma$. Then we say $n+1$ was inserted if the 0 -th place of $\sigma$ if $i=1$ and $j=0$. In fact, this will be the only place where we shall insert $n+1_{0}$ in our map. If $j \geq 1$, then we say that $n+1$ is inserted in $(j-1) n+i$-th place in $\sigma$. For example, if $\sigma=\left(1_{1}, 4_{2}, 3_{1}\right)\left(2_{3}, 7_{1}, 5_{3}\right)\left(6_{2}\right)$, then $\tau=\left(1_{1}, 8_{0}, 4_{2}, 3_{1}\right)\left(2_{3}, 7_{1}, 5_{3}\right)\left(6_{2}\right)$ is the result of inserting 8 into the 0 -th place in $\sigma$ and $\tau=\left(1_{1}, 4_{2}, 3_{1}\right)\left(2_{3}, 8_{3}, 7_{1}, 5_{3}\right)\left(6_{2}\right)$ is the result of inserting 8 in the $(3-1) 7+2=16$-th place in $\sigma$.

2. Given an element $\pi \in C S P t n_{n, k}$, suppose that $\delta \in C S P t n_{n+1, k}$ is the result of inserting the colored number $n+1_{j}$ into the $r$-th part of $\pi$ where we order the parts by the increasing smallest elements in $|\pi|$. Then we say $n+1$ was inserted in the 0 -th place of $\pi$ if $j=0$ and $r=1$. Again, this will be the only place where we shall insert $n+1_{0}$ in our map. If $j \geq 1$, then we say that $n+1$ is inserted in $(j-1) k+r$-th place in $\pi$. For example, if $\pi=\left\langle\left\{1_{1}, 4_{2}, 5_{1}\right\},\left\{2_{3}, 3_{1}, 6_{3}\right\},\left\{7_{2}\right\}\right\rangle$, then $\delta=\left\langle\left\{1_{1}, 4_{2}, 5_{1}, 8_{0}\right\},\left\{2_{3}, 3_{1}, 6_{3}\right\},\left\{7_{2}\right\}\right\rangle$ is the result of inserting 8 into the 0 -th place in $\pi$ and $\delta=\left\langle\left\{1_{1}, 4_{2}, 5_{1},\right\},\left\{2_{3}, 3_{1}, 6_{3},\right\},\left\{7_{2}, 8_{2}\right\}\right\rangle$ is the result of inserting 8 in the $(2-1) 3+3=6$-th place in $\pi$.

Let $U_{n, k}^{0}$ denote the set of pairs $(\sigma, \pi)$ where $\sigma$ is a colored permutation of $[n]$ and $\pi$ is a colored set partition of $[n]$ such that

1. $S M(\sigma)=S M(\pi)$, and

2. there are exactly $k$ elements $i \in S M(\sigma)=S M(\pi)$ which are colored 0 in both $\sigma$ and $\pi$.

Note that $U_{n, 0}^{0}$ is the set of pairs $(\sigma, \pi)$ where $\sigma$ is a colored permutation of $[n]$ and $\pi$ is a colored set partition of $[n]$ such that $S M(\sigma)=S M(\pi)$ and there are no $i \in S M(\sigma)=$ $S M(\pi)$ which are colored 0 in both $\sigma$ and $\pi$.

Next we define a map $\Gamma_{n, k}^{\mathcal{T}, \mathcal{S}}: \mathcal{N}_{n-k}\left(\mathcal{B}^{\mathcal{A}}\right) \rightarrow U_{n, k}^{0}$. Given $\mathbb{P} \in \mathcal{N}_{n-k}\left(\mathcal{B}^{\mathcal{A}}\right)$, we construct $\Gamma_{n, k}^{\mathcal{T}, \mathcal{S}}(\mathbb{P})=(\sigma, \pi)$ by constructing a sequence of pairs $\left(\sigma^{(1)}, \pi^{(1)}\right), \ldots,\left(\sigma^{(n)}, \pi^{(n)}\right)=(\sigma, \pi)$ in stages as follows.

Stage 1. We have three cases.

Case I. There is no rook in the first column of $\mathbb{P}$. Then $\sigma^{(1)}=\left(1_{0}\right)$ and $\pi^{(1)}=\left\{1_{0}\right\}$. Thus $\left(\sigma^{(1)}, \pi^{(1)}\right) \in U_{1,1}^{0}$.

Case II. There is a rook in the first column in the $i$-th square of the augmented part of $\mathcal{B}^{\mathcal{A}}$ reading from bottom to top. Then $\sigma^{(1)}=\left(1_{0}\right)$ and $\pi^{(1)}=\left\{1_{i}\right\}$. Thus $\left(\sigma^{(1)}, \pi^{(1)}\right) \in U_{1,0}^{0}$. 


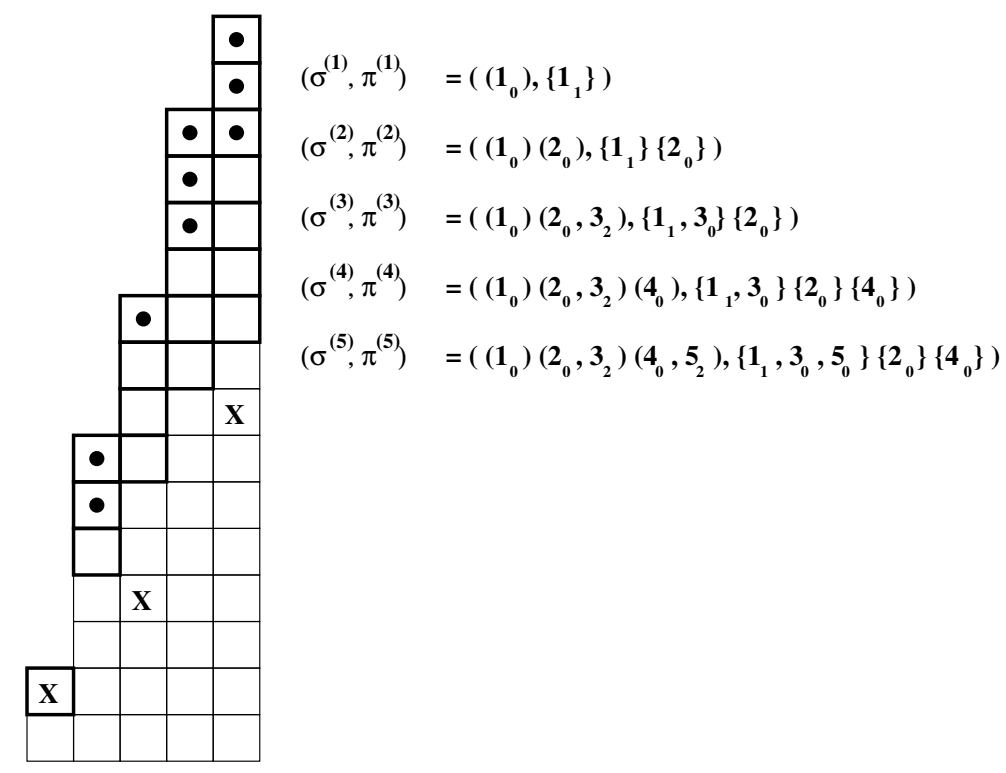

Figure 9: An example of the map $\Gamma$.

Case III. There is a rook in the first column in the $i$-th square of the base part of $\mathcal{B}^{\mathcal{A}}$ reading from bottom to top. Then $\sigma^{(1)}=\left(1_{i}\right)$ and $\pi^{(1)}=\left\{1_{0}\right\}$. Thus $\left(\sigma^{(1)}, \pi^{(1)}\right) \in U_{1,0}^{0}$.

Stage s+1. Assume we have constructed $\left(\sigma^{(s)}, \pi^{(s)}\right)$ so that if $\mathbb{P}$ has $s-r$ rooks in the first $r$ columns, then $\left(\sigma^{(s)}, \pi^{(s)}\right) \in U_{s, r}^{0}$. Then again we have three cases.

Case I. There is no rook in the $(s+1)$-st column of $\mathbb{P}$. Then $\sigma^{(s+1)}$ is the result of adding a new cycle $\left((s+1)_{0}\right)$ to $\sigma^{(s)}$ and $\pi^{(s+1)}$ is the result of adding a new part $\left\{(s+1)_{0}\right\}$ to $\pi^{(s)}$.

Case II. There is a rook in the $(s+1)$-st column in the $i$-th square of the augmented part of $\mathcal{B}^{\mathcal{A}}$ reading from bottom to top. Then $\sigma^{(s+1)}$ is the result of inserting $s+1$ in the 0 -th position of $\sigma^{(s)}$ and $\pi^{(s+1)}$ is the result of inserting $s+1$ in the $i$-th position of $\pi^{(s)}$.

Case III. There is a rook in the $(s+1)$-st column in the $i$-th square of the base part of $\mathcal{B}^{\mathcal{A}}$ reading from bottom to top. Then $\sigma^{(s+1)}$ is the result of inserting $s+1$ in the $i$-th position of $\sigma^{(s)}$ and $\pi^{(s+1)}$ is the result of inserting $s+1$ in the 0 -th position of $\pi^{(s)}$.

For example, suppose that $n=5, \mathcal{S}=(1,2,1,2,1)$, and $\mathcal{T}=(1,3,2,2,1)$. Then in Figure 9 , we have pictured a placement $\mathbb{P} \in \mathcal{N}_{3}\left(\mathcal{B}^{\mathcal{A}}\right)$ and its corresponding sequence $\left(\sigma^{(1)}, \pi^{(1)}\right), \ldots,\left(\sigma^{(5)}, \pi^{(5)}\right)=(\sigma, \pi)$.

Note that $\sigma^{(i)}$ is the permutation whose cycle structure is constructed from $\sigma$ by removing all elements with absolute value greater than $i$ and $\pi^{(i)}$ is the set partition of $[i]$ which is the result of removing all elements with absolute value greater than $i$ in $\pi$. 
Accordingly, given a pair $(\sigma, \pi)$ in the range of $\Gamma_{n, k}^{\mathcal{T}, \mathcal{S}}$, we can reconstruct the sequence

$$
\left(\sigma^{(1)}, \pi^{(1)}\right), \ldots,\left(\sigma^{(n)}, \pi^{(n)}\right)=(\sigma, \pi) .
$$

Going the other way, given the sequence $\left(\sigma^{(1)}, \pi^{(1)}\right), \ldots,\left(\sigma^{(n)}, \pi^{(n)}\right)$, we can decode which case of the above construction was used at each stage so that we can reconstruct the placement $\mathbb{P}$ such that $\Gamma_{n, k}^{\mathcal{T}, \mathcal{S}}(\mathbb{P})=(\sigma, \tau)$. Thus $\Gamma_{n, k}^{\mathcal{T}, \mathcal{S}}$ is one-to-one. Moreover, it is not difficult to characterize the range of $\Gamma_{n, k}^{\mathcal{T}, \mathcal{S}}$. That is, $(\sigma, \pi)$ is in the range of $\Gamma_{n, k}^{\mathcal{T}, \mathcal{S}}$ if and only if

1. $(\sigma, \pi) \in U_{n, k}^{0}$ and for all $1 \leq i<n$, the number of cycles of $\sigma^{(i)}$ equals the number of parts of $\pi^{(i)}$,

2. $S M(\sigma)=S M(\pi)$,

3. either (a) 1 is colored 0 in both $\sigma$ and $\pi$, (b) 1 is colored $s$ for some $1 \leq s \leq T_{1}$ in $\sigma$ and 1 is colored 0 in $\pi$, or 1 is colored $s$ for some $1 \leq s \leq S_{1}$ in $\pi$ and 1 is colored 0 in $\sigma$,

4. if $i>1$, then $i \in S M(\sigma)=S M(\pi)$ if and only if $i$ is colored 0 in $\sigma$ and $\pi$ so that $\sigma$ has $k$ cycles and $\pi$ has $k$ parts if 1 is colored 0 in both $\sigma$ and $\pi$ and $\sigma$ has $k+1$ cycles and $\pi$ has $k+1$ parts if 1 is not colored 0 in both $\sigma$ and $\pi$,

5. if $i>1, i \notin S M(\pi), \pi^{(i-1)}$ has $r$ parts, and $i$ is inserted in position $s$ in $\pi^{(i-1)}$ where $s>0$, then $i$ is inserted in position 0 in $\sigma$ and $1 \leq s \leq S_{r+1}$ if 1 has color 0 in both $\sigma$ and $\pi$ and $1 \leq s \leq S_{r}$ if 1 does not have color 0 in both $\sigma$ and $\pi$, and

6. if $i>1, i \notin S M(\sigma)$, and $i$ is inserted in position $s$ in $\sigma^{(i-1)}$ where $s>0$, then $i$ is inserted in position 0 in $\pi$ and $1 \leq s \leq T_{i}$.

Here for condition (5.), note that if the first column of $\mathbb{P}$ is empty so that 1 is colored 0 in both $\sigma$ and $\pi$, then when $\pi^{(i-1)}$ has $r$ parts, there must be $r$ empty columns in the first $i-1$ columns of $\mathbb{P}$. Hence there are $i-1-r$ rooks in the first $i$ columns of $\mathbb{P}$ and these rooks cancel the $s_{m}$-th part of the augmented part of the board for $m=i, i-$ $1, \ldots, i-(i-1-r-1)=r+2$. So, we can only put rooks in the first $s_{1}+\cdots+s_{r+1}=S_{r+1}$ cells in column $i$. Similarly, if there is a rook in the first column of $\mathbb{P}$ so that it cannot be the case that 1 is colored 0 in both $\sigma$ and $\pi$, then when $\pi^{(i-1)}$ has $r$ parts, there must be $r-1$ empty columns in the first $i-1$ columns of $\mathbb{P}$. Hence there are $i-1-(r-1)$ rooks in the first $i$ columns of $\mathbb{P}$ and these rooks cancel the $s_{m}$-th part of the augmented part of the board for $m=i, i-1, \ldots, i-(i-r-1)=r+1$. Thus, we can only put rooks in the first $s_{1}+\cdots+s_{r}=S_{r}$ cells in column $i$. This given, we let $\mathcal{H}_{n, k}^{\mathcal{T}, \mathcal{S}}$ denote the set of all pairs $(\sigma, \pi) \in U_{n, k}^{0}$ satisfying the six conditions above. Thus, $\Gamma_{n, k}^{\mathcal{T}, \mathcal{S}}$ is a bijection from $\mathcal{N}_{n-k}\left(\mathcal{B}^{\mathcal{A}}\right)$ onto $\mathcal{H}_{n, k}^{\mathcal{T}, \mathcal{S}}$.

Given $(\sigma, \pi) \in \mathcal{H}_{n, k}^{\mathcal{T}, \mathcal{S}}$, let $\sigma^{(i)}$ be the permutation whose cycle structure is the result of eliminating the elements of absolute value greater than $i$ in the cycle structure of 
$\sigma$. Similarly, let $\pi^{(i)}$ be the set partition that is the result of eliminating the elements of absolute value greater than $i$ in $\pi$. Then if $\left(\sigma^{(1)}, \pi^{(1)}\right)=\left(\left(1_{s}\right),\left\langle\left\{1_{t}\right\}\right\rangle\right.$, then we define the weight of 1 in $(\sigma, \pi), W_{(\sigma, \pi)}(1)$, to be $s+t$. If $1<k \leq n$, then we define the weight of $k$ in $(\sigma, \pi), W_{(\sigma, \pi)}(k)$, to be $s+t$ where $k$ is inserted in $s$-th position in $\sigma^{(k-1)}$ and $k$ is inserted in the $t$-th position in $\pi^{(k-1)}$. We then define the weight of $(\sigma, \pi), W(\sigma, \pi)$, by setting

$$
W(\sigma, \pi)=\prod_{i=1}^{n} q^{W_{(\sigma, \pi)}(i)},
$$

and it is straightforward to check that we have defined the map $\Gamma_{n, k}^{\mathcal{T}, \mathcal{S}}$ so that if $\Gamma_{n, k}^{\mathcal{T}, \mathcal{S}}(\mathbb{P})=$ $(\sigma, \pi)$, then

$$
\nu_{q, \mathcal{B}^{\mathcal{A}}}(\mathbb{P})=W(\sigma, \pi) .
$$

Finally, we observe that if $(\sigma, \pi) \in \mathcal{H}_{n, k}^{\mathcal{T}, \mathcal{S}}$ and $\Gamma_{n, k}^{\mathcal{T}, \mathcal{S}}(\mathbb{P})=(\sigma, \pi)$, then the number of rooks of $\mathbb{P}$ which lie in the base part of $\mathcal{B}^{\mathcal{A}}$ is $i$, such that $i$ is inserted in position $s$ in $\sigma^{(i-1)}$ where $s>0$. Similarly, the number of rooks of $\mathbb{P}$ which lie in the augmented part of $\mathcal{B}^{\mathcal{A}}$ is $i$, such that $i$ is inserted in position $s$ in $\pi^{(i-1)}$ where $s>0$. Let $\operatorname{Pos}(\sigma)$ and $\operatorname{Pos}(\pi)$ equal the number of $i$ such that $i$ is inserted in position $s$ in $\sigma^{(i-1)}$ or $\pi^{(i-1)}$ respectively, where $s>0$. It then immediately follows from our combinatorial interpretations $r_{n, k}^{\mathcal{T} \uparrow, \mathcal{S} \downarrow}(q), r_{n, k}^{\mathcal{T} \downarrow, \mathcal{S} \uparrow}(q), r_{n, k}^{\mathcal{T} \uparrow, \mathcal{S} \uparrow}(q)$, and $r_{n, k}^{\mathcal{T} \downarrow, \mathcal{S} \uparrow}(q)$ in terms of $r_{n-k}\left(\mathcal{B}^{\mathcal{A}}\right)$ given in (3.3), (3.5), (3.7), and (3.11) that we have the following theorem.

Theorem 4.1. For any sequences $\mathcal{S}=\left(s_{1}, s_{2}, \ldots\right)$ and $\mathcal{T}=\left(t_{1}, t_{2}, \ldots\right)$ of non-negative integers,

$$
\begin{aligned}
& \text { 1. } r_{n, k}^{\mathcal{T}, \mathcal{S} \downarrow}(q)=\sum_{(\sigma, \pi) \in \mathcal{H}_{n, k}^{\mathcal{T}, \mathcal{S}}} W(\sigma, \pi), \\
& \text { 2. } r_{n, k}^{\mathcal{T} \downarrow \mathcal{S} \uparrow}(q)=(-1)^{n-k} \sum_{(\sigma, \pi) \in \mathcal{H}_{n, k}^{\mathcal{T}, \mathcal{S}}} W(\sigma, \pi), \\
& \text { 3. } r_{n, k}^{\mathcal{T} \uparrow, \mathcal{S} \uparrow}(q)=\sum_{(\sigma, \pi) \in \mathcal{H}_{n, k}^{\mathcal{T}, \mathcal{S}}}(-1)^{\operatorname{Pos}(\pi)} W(\sigma, \pi), \text { and } \\
& \text { 4. } r_{n, k}^{\mathcal{T} \downarrow, \mathcal{S} \downarrow}(q)=\sum_{(\sigma, \pi) \in \mathcal{H}_{n, k}^{\mathcal{T}, \mathcal{S}}}(-1)^{\operatorname{Pos}(\sigma)} W(\sigma, \pi) .
\end{aligned}
$$

We note that if $\mathcal{S}=(0,0,0, \ldots)$, then it must be the case that for all $(\sigma, \pi) \in \mathcal{H}_{n, k}^{\mathcal{T}, \mathcal{S}}$, $\operatorname{Pos}(\pi)=0$. This means that if $(\sigma, \pi) \in \mathcal{H}_{n, k}^{\mathcal{T}, \mathcal{S}}$, then $\pi$ is completely determined by $\sigma$. That is, we are forced to have $S M(\sigma)=S M(\pi)$ and every element of $\pi$ must be colored with 0 . If $i \notin S M(\pi)$, then the element $i_{0}$ must lie in the first part of $\pi$. For example, if $\sigma=\left(1_{1}, 4_{2}, 3_{1}\right)\left(2_{0}, 8_{2}, 7_{1}, 5_{3}\right)\left(6_{0}\right)$, then it must be the case that $\pi=$ $\left\langle\left\{1_{0}, 3_{0}, 4_{0}, 5_{0}, 7_{0}, 8_{0}\right\},\left\{2_{0}\right\},\left\{6_{0}\right\}\right\rangle$. Thus, in such a situation, $\pi$ carries no information nor does it contribute to $W(\sigma, \pi)$, and so we are reduced to considering only colored 
permutations with certain restricted insertion patterns. That is, let $\mathcal{G}_{n, k}^{\mathcal{T}}$ denote the set of colored permutations $\sigma$ of $[n]$ such that

1. exactly $k$ elements of $S M(\sigma)$ are colored 0 ,

2. 1 is colored $s$ for some $0 \leq s \leq T_{1}$,

3. if $i \in S M(\sigma)-\{1\}$, then $i$ is colored with 0 so that $\sigma$ has $k$ cycles if 1 is colored 0 and $\sigma$ has $k+1$ cycles if 1 is not colored 0 , and

4. if $i>1$ and $i \notin S M(\sigma)$, then $i$ is inserted in position $s$ in $\sigma^{(i-1)}$ where $1 \leq s \leq T_{i}$.

This gives us the following corollary.

Corollary 1. If $\mathcal{S}=(0,0,0, \ldots)$ and $\mathcal{T}=\left(t_{1}, t_{2}, t_{3}, \ldots\right)$ is a sequence of non-negative integers, then

$$
\begin{aligned}
& \text { 1. } r_{n, k}^{\mathcal{T}, \mathcal{S} \downarrow}(q)=r_{n, k}^{\mathcal{T \uparrow} \mathcal{S} \uparrow}(q)=\sum_{\sigma \in \mathcal{G}_{n, k}^{\mathcal{T}}} W(\sigma), \text { and } \\
& \text { 2. } r_{n, k}^{\mathcal{T} \downarrow \mathcal{S} \downarrow}(q)=r_{n, k}^{\mathcal{T} \downarrow \mathcal{S} \uparrow}(q)=(-1)^{n-k} \sum_{\sigma \in \mathcal{G}_{n, k}^{\mathcal{T}}} W(\sigma),
\end{aligned}
$$

where $W(\sigma)=a_{1}+\cdots+a_{n}$ if the color of 1 is $a_{1}$ and $i$ is inserted into position $a_{i}$ in $\sigma^{(i-1)}$ for $i=2, \ldots, n$.

It is easy to check that if, in addition, $T_{i} \leq i-1$ for all $i$, then it will be the case that the color of 1 is 0 and the color of any $i \notin S M(\sigma)$ will be 1 . Thus the minimal elements of $S M(\sigma)$ must have color 0 and all other elements of $\sigma$ must have color 1. Clearly, in such a situation, the colors on the elements of $\sigma$ are completely determined so that we can just drop the colors. It then follows that the corresponding rook numbers can be described by $q$-counting permutations with certain restricted cycle structures. That is, given any permutation $\sigma \in S_{n}$, organize the cycles of $\sigma$ so that the smallest element of each cycle is the leftmost. Now, for any $1 \leq i \leq n$, define $c_{\sigma}(i)=0$ if $i$ is the smallest element in its cycle and if $i$ is not the smallest element in its cycle, define $c_{\sigma}(i)$ as the rightmost element in the cycle containing $i$ that is both to the left of $i$ and smaller than $i$. Note that in the latter case, this means $i$ was inserted in position $c_{\sigma}(i)$ according to our definitions. We then let $R P e r m_{n, k}^{\mathcal{T}}$ denote the set of permutations $\sigma \in S_{n}$ with $k$ cycles such that for all $1 \leq i \leq n$, either $i$ is the smallest element in its cycle or $1 \leq c_{\sigma}(i) \leq T_{i}$ if $i$ is not the smallest element in its cycle. Then we have the following corollary.

Corollary 2. If $\mathcal{S}=(0,0,0, \ldots)$ and $\mathcal{T}=\left(t_{1}, t_{2}, t_{3}, \ldots\right)$ is a sequence of non-negative integers such that for all $i, t_{1}+\cdots+t_{i} \leq i-1$, then

$$
\text { 1. } r_{n, k}^{\mathcal{T \uparrow}, \mathcal{S} \downarrow}(q)=r_{n, k}^{\mathcal{T} \uparrow, \mathcal{S} \uparrow}(q)=\sum_{\sigma \in R P \operatorname{Perm}}^{\tau, k} \mathcal{T} W(\sigma) \text {, and }
$$




$$
\text { 2. } r_{n, k}^{\mathcal{T} \downarrow, \mathcal{S} \downarrow}(q)=r_{n, k}^{\mathcal{T} \downarrow, \mathcal{S} \uparrow}(q)=(-1)^{n-k} \sum_{\sigma \in R P e r m_{n, k}^{\mathcal{T}}} W(\sigma),
$$

where $W(\sigma)=\sum_{i=1}^{n} c_{\sigma}(i)$.

For example, suppose that $\mathcal{T}=(0,1,0,1,0,1, \ldots)$ so that $T_{1}=0$ and for $i \geq 1$, $T_{2 i}=T_{2 i+1}=i$. Then $\operatorname{RPerm}_{n, k}^{\mathcal{T}}$ is the set of all permutations $\sigma \in S_{n}$ such that $\sigma$ has $k$ cycles and for all $i, c_{\sigma}(i) \leq\left\lfloor\frac{i}{2}\right\rfloor$ if $i$ is not the smallest element in its cycle.

We can make a similar argument if $\mathcal{T}=(0,0,0, \ldots)$. In this situation, it must be the case that for all $(\sigma, \pi) \in \mathcal{H}_{n, k}^{\mathcal{T}, \mathcal{S}}, \operatorname{Pos}(\sigma)=0$. This means that if $(\sigma, \pi) \in \mathcal{H}_{n, k}^{\mathcal{T}, \mathcal{S}}$, then $\sigma$ is completely determined by $\pi$. That is, we are forced to have $S M(\sigma)=S M(\pi)$ and every element of $\sigma$ must be colored with 0 . So if $i \notin S M(\sigma)$, then the element $i_{0}$ must be inserted directly after 1 in $\sigma^{(i-1)}$. This will force the first cycle of $\sigma$ to consist of the element $1_{0}$ followed by all elements of the set $[n]-S M(\pi)$ in decreasing order colored by 0 . For example, if $\pi=\left\langle\left\{1_{1}, 3_{2}, 4_{1}\right\},\left\{2_{0}, 5_{2}, 8_{1}\right\},\left\{6_{0}, 7_{2}\right\}\right\rangle$, then it must be the case that $\sigma=\left(1_{0}, 8_{0}, 7_{0}, 5_{0}, 4_{0}, 3_{0}\right)\left(2_{0}\right)\left(6_{0}\right)$. Thus, in such a situation, $\sigma$ carries no information nor does it contribute to $W(\sigma, \pi)$, and so we are reduced to considering only colored set partitions with certain restricted insertion patterns. That is, let $\mathcal{K}_{n, k}^{\mathcal{T}}$ denote the set of colored set partitions $\pi$ of $[n]$ such that

1. exactly $k$ elements of $S M(\pi)$ are colored 0 ,

2. 1 is colored $s$ for some $0 \leq s \leq S_{1}$,

3. if $i \in S M(\pi)-\{1\}$, then $i$ is colored with 0 so that $\pi$ has $k$ parts if 1 is colored 0 and $\pi$ has $k+1$ parts if 1 is not colored with 0 , and

4. if $i>1, i \notin S M(\pi)$, and $\pi^{(i-1)}$ has $r$ parts, then $i$ is inserted in position $s$ in $\pi^{(i-1)}$ where $1 \leq s \leq S_{r+1}$ if 1 is colored with 0 in $\pi$ and where $1 \leq s \leq S_{r}$ if 1 is not colored 0 in $\pi$.

This gives us the following corollary.

Corollary 3. If $\mathcal{T}=(0,0,0, \ldots)$ and $\mathcal{S}=\left(s_{1}, s_{2}, s_{3}, \ldots\right)$ is a sequence of non-negative integers, then

$$
\begin{aligned}
& \text { 1. } r_{n, k}^{\mathcal{T \uparrow}, \mathcal{S} \downarrow}(q)=r_{n, k}^{\mathcal{T} \downarrow, \mathcal{S} \downarrow}(q)=\sum_{\pi \in \mathcal{K}_{n, k}^{\mathcal{S}}} W(\pi) \text {, and } \\
& \text { 2. } r_{n, k}^{\mathcal{T} \uparrow, \mathcal{S} \uparrow}(q)=r_{n, k}^{\mathcal{T} \downarrow, \mathcal{S} \uparrow}(q)=(-1)^{n-k} \sum_{\pi \in \mathcal{K}_{n, k}^{\mathcal{T}}} W(\pi),
\end{aligned}
$$

where $W(\pi)=a_{1}+\cdots+a_{n}$ if the color of 1 is $a_{1}$ and $i$ is inserted into position $a_{i}$ in $\pi^{(i-1)}$ for $i=2, \ldots, n$. 
If, in addition, we assume that $S_{i} \leq i-1$ for all $i$, then it will be the case that the color of 1 is 0 in both $\sigma$ and $\pi$ since $s_{1}=0$ in that case. It follows that if $\pi^{(i-1)}$ has $r$ parts, then we must place $i$ in one of the positions $1, \ldots, S_{r+1}=r$ if $i$ does not start a new part in $\pi$. But since we have $r$ parts in which to place $i$, we can always do this with an element colored 1. Thus the minimal elements of $S M(\pi)$ must have color 0 and all other elements of $\pi$ must have color 1. Clearly, in such a situation, the colors of the elements of $\pi$ are completely determined so that we can just drop the colors. It then follows that the corresponding rook numbers can be described as counting set partitions with certain restricted part structures. For any $i$, we define $c_{\pi}(i)=0$ if $i$ is the smallest element in its part. If $i$ is not the smallest element in its part, we define $c_{\pi}(i)=j$ when $i$ is in the $j$-th part of $\pi$. We then let $R S P t n_{n, k}^{\mathcal{S}}$ denote the set of all set partitions of $[n]$ with $k$ parts such that for all $i=1, \ldots, n$, either $i$ is the smallest element in its part or $1 \leq c_{\pi}(i) \leq S_{r+1}$ if $i$ is not the smallest element in its cycle and $\pi^{(i+1)}$ has $r$ parts. Then we have the following corollary.

Corollary 4. If $\mathcal{T}=(0,0,0, \ldots)$ and $\mathcal{S}=\left(s_{1}, s_{2}, s_{3}, \ldots\right)$ is a sequence of non-negative integers such that for all $i, s_{1}+\cdots+s_{i} \leq i-1$, then

$$
\begin{aligned}
& \text { 1. } r_{n, k}^{\mathcal{T} \uparrow, \mathcal{S} \downarrow}(q)=r_{n, k}^{\mathcal{T} \downarrow, \mathcal{S} \downarrow}(q)=\sum_{\pi \in R S P t n_{n, k}^{\mathcal{S}}} W(\pi) \text {, and } \\
& \text { 2. } r_{n, k}^{\mathcal{T} \downarrow, \mathcal{S} \uparrow}(q)=r_{n, k}^{\mathcal{T} \uparrow, \mathcal{S} \uparrow}(q)=(-1)^{n-k} \sum_{\pi \in R S P t n_{n, k}^{\mathcal{S}}} W(\pi),
\end{aligned}
$$

where $W(\pi)=\sum_{i=1}^{n} c_{\pi}(i)$.

\section{References}

[1] K. S. Briggs and J. B. Remmel, A $p, q$-analogue of a formula of Frobenius, Electron. J. Combin. 10(1) (2003), \#R9.

[2] K. S. Briggs and J. B. Remmel, $m$-Rook numbers and a generalization of a formula of Frobenius to $\mathcal{C}_{m} \S \mathcal{S}_{n}$, J. Combin. Theory Ser. A 113 (2006), 1138-1171

[3] A. M. Garsia and J. B. Remmel, $Q$-counting rook configurations and a formula of Frobenius, J. Combin. Theory Ser. A 41 (1986), 246-275.

[4] J. Goldman and J. Haglund Generalized rook polynomials, J. Combin. Theory Ser. A 91 (2000), 509-530.

[5] H. W. Gould, The $q$-Stirling numbers of the first and second kinds, Duke Math. J. 28 (1961), 281-289.

[6] J. Lindsay, T. Mansour, and M Shattuck, A new combinatorial interpretation of a $q$-analogue of the Lah numbers, J. Comb. 2:2 (2011), 245-264. 
[7] A. de Médicis and P. Leroux, A unified combinatorial approach for $q-(\& p, q-)$ Stirling numbers, J. Statist. Plann. Inference 34 (1993), 89-105.

[8] A. de Médicis and P. Leroux, Generalized Stirling numbers, convolution formulae and $p, q$-analogues, Canad. J. Math. 47 (1995), 474-499.

[9] B. K. Miceli, Two $q$-analogues of poly-Stirling numbers, J. Integer Seq. 14 (2011), 11.9.6

[10] B. K. Miceli and J. Remmel, Augmented rook boards and general product formulas, Electron. J. Combin. 15 (2008), R85.

[11] S. C. Milne, Restricted growth functions, rank row matchings of partition lattices, and $q$-Stirling numbers, Adv. in Math. 43 (1982), 173-196.

[12] J. B. Remmel and M. Wachs, Rook theory, generalized Stirling numbers and $(p, q)$ analogues, Electron. J. Combin. 11 (2004), R84.

[13] M. Wachs and D. White, $p, q$-Stirling numbers and set partition statistics, J. Combin. Theory Ser. A 56 (1991), 27-46.

[14] M. Wachs, $\sigma$-Restricted growth functions and $p, q$-Stirling numbers, J. Combin. Theory Ser. A 68 (1994), 470-480.

[15] D. White, Interpolating set partition statistics, J. Combin. Theory Ser. A 68 (1994), 262-295. 